\title{
Turbulence characterization from a forward-looking nacelle lidar
}

\author{
Alfredo Peña, Jakob Mann, and Nikolay Dimitrov \\ DTU Wind Energy, Technical University of Denmark, Roskilde, Denmark \\ Correspondence to: Alfredo Peña (aldi@dtu.dk)
}

Received: 13 December 2016 - Discussion started: 19 December 2016

Revised: 17 February 2017 - Accepted: 21 February 2017 - Published: 13 March 2017

\begin{abstract}
We present two methods to characterize turbulence in the turbine inflow using radial velocity measurements from nacelle-mounted lidars. The first uses a model of the three-dimensional spectral velocity tensor combined with a model of the spatial radial velocity averaging of the lidars, and the second uses the ensembleaveraged Doppler radial velocity spectrum. With the former, filtered turbulence estimates can be predicted, whereas the latter model-free method allows us to estimate unfiltered turbulence measures. Two types of forwardlooking nacelle lidars are investigated: a pulsed system that uses a five-beam configuration and a continuouswave system that scans conically. For both types of lidars, we show how the radial velocity spectra of the lidar beams are influenced by turbulence characteristics, and how to extract the velocity-tensor parameters that are useful to predict the loads on a turbine. We also show how the velocity-component variances and co-variances can be estimated from the radial-velocity unfiltered variances of the lidar beams. We demonstrate the methods using measurements from an experiment conducted at the Nørrekær Enge wind farm in northern Denmark, where both types of lidars were installed on the nacelle of a wind turbine. Comparison of the lidar-based along-wind unfiltered variances with those from a cup anemometer installed on a meteorological mast close to the turbine shows a bias of just $2 \%$. The ratios of the unfiltered and filtered radial velocity variances of the lidar beams to the cup-anemometer variances are well predicted by the spectral model. However, other lidar-derived estimates of velocity-component variances and co-variances do not agree with those from a sonic anemometer on the mast, which we mostly attribute to the small cone angle of the lidar. The velocity-tensor parameters derived from sonic-anemometer velocity spectra and those derived from lidar radial velocity spectra agree well under both near-neutral atmospheric stability and high wind-speed conditions, with differences increasing with decreasing wind speed and increasing stability. We also partly attribute these differences to the lidar beam configuration.
\end{abstract}

\section{Introduction}

Recently, lidars have been mounted on the nacelle of wind turbines to investigate wake characteristics (Bingöl et al., 2010; Machefaux et al., 2016; Trujillo et al., 2016) and today are extensively used in a forward-looking (FL) mode to scan the turbine inflow for many purposes. One of such is power-performance measurements; FL nacelle lidars decrease the statistical uncertainty of the measured power curve when compared to that based on mast measurements (Wagner et al., 2014). The statistical uncertainty associated with load validation can potentially also be reduced (Dimitrov and Natarajan, 2016). Another important use of FL nacelle li- dars is turbine control; they have the potential to reduce loads and increase energy capture (Mikkelsen et al., 2013; Schlipf et al., 2015). Irrespectively of the application, FL nacelle lidars are primarily aimed to characterize the inflow in front of the turbine. Inflow characterization has been performed using lidars of different types and configurations for several years (Hardesty et al., 1981; Peña et al., 2010b; Aitken et al., 2012). However, FL nacelle lidars have the advantage of measuring the inflow in front of the turbines more "effectively" than other types of lidars because they scan over the area in front of the turbine and yaw with it. Therefore, they can potentially be used for measuring the yaw misalignment of wind tur- 
bines (Fleming et al., 2014). If they become widely applied in the wind-energy industry, they could be used to characterize wind resources in regions where measurements from meteorological towers are scarce or non-existent.

Similar to ground-based lidars, there are two main types of FL nacelle lidars, pulsed and continuous-wave (CW), which mainly differ, for the purpose of turbulence estimation, on the measurement probe volume and the scanning strategies (specific details are given later). As with any other Doppler lidar, they only measure the radial velocity along the laser beam or line-of-sight velocity. As their measurement probe volumes are generally larger than those of cup and sonic anemometers, they might not be able to measure small turbulent eddies, which leads to "filtered" turbulence estimates; however, as they scan the atmosphere with laser beams in different directions, there might be contributions (contamination) from different velocity components that can lead, for some scanning configurations and under certain turbulence conditions, to turbulence estimates that might be even higher than those from cup or sonic anemometers. A detailed analysis on how lidar-based turbulence estimates can be assessed, filtered, and contaminated is presented in Sathe and Mann (2013).

Here we use time series of radial velocity measurements from different beams emitted by a FL nacelle lidar to estimate the turbulence parameters of the three-dimensional spectral velocity tensor model by Mann (1994) (hereafter the Mann model). This model is chosen because it fits the atmospheric-turbulence velocity spectra for different surface, wind, and atmospheric-stability conditions within the first $\approx 100 \mathrm{~m}$ from the ground well (Peña et al., 2010a; Chougule et al., 2015) and is widely used to perform aeroelastic simulations of wind turbines. The ultimate objective of this study is to find out whether nacelle lidars can be used independently (i.e., without the need of extra measurements, e.g., from instruments on meteorological masts) to extract turbulence information from the inflow. Nacelle lidars can potentially infer the inflow characteristics that actually impact the turbines better than traditional nacelle or mast anemometry because they can scan over an air volume, which is more representative of the flow entering the rotor plane. We also use, when possible, information of the Doppler radial velocity spectrum to estimate the "unfiltered" lidar beam variances and, from those, we estimate the velocity-component variances and covariances (Mann et al., 2010).

This paper is organized as follows. In Sect. 2, we introduce shortly the characteristics of the wind field, how this is represented by the Mann model, and how to extract the turbulence characteristics from velocity spectra. Section 3 shows the two types of nacelle lidars investigated here, Sect. 3.1 illustrates how to derive the radial velocity spectra from the different lidar configurations and how these spectra are influenced by both the lidar configuration and the turbulence characteristics of the Mann model, and Sect. 3.2 shows how to extract turbulence information from the lidars' radial velocity spectra. Section 4 introduces the Nørrekær Enge wind farm and the measurements of the experimental campaign. Section 5 provides the details on the way the measurements are analyzed, and Sect. 6 shows the comparison of turbulence characteristics extracted from nacelle-lidar measurements and those from sonic- and cup-anemometer measurements. Finally, we provide some discussion and conclusions in the last two sections.

\section{Turbulence background}

The wind field is described by a vector field $\boldsymbol{u}(\boldsymbol{x})$, where the time argument is eliminated because Taylor's frozen turbulence hypothesis is assumed (Mizuno and Panofsky, 1975) and $\boldsymbol{x}$ is the position vector in space, $\boldsymbol{x}=(x, y, z)$. The mean value of the homogeneous velocity field is $\langle\boldsymbol{u}(\boldsymbol{x})\rangle=(U, 0,0)$, so the coordinate $x$ is in the mean wind direction. The wind field can also be written as a Fourier integral,

$$
\begin{aligned}
\boldsymbol{u}(\boldsymbol{x}) & =\int \boldsymbol{u}(\boldsymbol{k}) e^{i \boldsymbol{k} \cdot \boldsymbol{x}} \mathrm{d} \boldsymbol{k} \Leftrightarrow \boldsymbol{u}(\boldsymbol{k}) \\
& =\frac{1}{(2 \pi)^{3}} \int \boldsymbol{u}(\boldsymbol{x}) e^{-i \boldsymbol{k} \cdot \boldsymbol{x}} \mathrm{d} \boldsymbol{x},
\end{aligned}
$$

where $\boldsymbol{k}$ is the wave vector (Batchelor, 1953; Mann, 1994). The ensemble average of the absolute squared Fourier coefficients is the spectral tensor:

$\left\langle u_{i}^{*}(\boldsymbol{k}) u_{j}\left(\boldsymbol{k}^{\prime}\right)\right\rangle=\Phi_{i j}(\boldsymbol{k}) \delta\left(\boldsymbol{k}-\boldsymbol{k}^{\prime}\right)$.

The spectral velocity tensor, $\Phi_{i j}$, is assumed to be described by the Mann model, which, besides $\boldsymbol{k}$, only contains three parameters: $\alpha \varepsilon^{2 / 3}, L$, and $\Gamma$, where $\alpha$ is the spectral Kolmogorov constant, $\varepsilon$ the specific rate of destruction of turbulent kinetic energy, $L$ a length scale related to the size of the turbulent eddies, and $\Gamma$ a parameter describing the anisotropy of the turbulence. From the spectral tensor, the one-point spectra are calculated by

$F_{i j}\left(k_{1}\right)=\iint \Phi_{i j}(\boldsymbol{k}) \mathrm{d} k_{2} \mathrm{~d} k_{3}$,

and typically, the three auto-spectra of the $u, v$, and $w$ components of the wind velocity, $F_{11}, F_{22}$, and $F_{33}$, respectively, together with the one-point cross-spectrum, $F_{13}$, are fitted simultaneously to measured or theoretical spectra in order to obtain the Mann-model parameters (hereafter referred to as Mann parameters). This procedure is described in Mann (1994). In order to facilitate the fitting, a two-parameter lookup table (LUT) with values of $F_{i j}\left(k_{1}\right)=F_{i j}\left(k_{1} ; \alpha \varepsilon^{2 / 3}=\right.$ $1, L=1, \Gamma)$ is precomputed. The mathematical identity

$F_{i j}\left(k_{1} ; \alpha \varepsilon^{2 / 3}, L, \Gamma\right)=L^{5 / 3} \alpha \varepsilon^{2 / 3} F_{i j}\left(k_{1} L ; 1,1, \Gamma\right)$

is used to calculate the spectra for arbitrary values of $k_{1}$, $\alpha \varepsilon^{2 / 3}, L$, and $\Gamma$. 


\section{Nacelle lidars}

Two types of FL nacelle lidars are investigated: a CW and a pulsed lidar. The lidars are assumed to be mounted close to the center of the rotor with $N$ beams pointing in different directions (see Fig. 1). For the CW lidar studied here, the beams point on a cone with the symmetry axis pointing upstream; Fig. 1's left panel shows a configuration with an arbitrary number of beams, $N=13$, of which 12 beams draw a conical surface and 1 is perpendicular to the rotor plane. The half opening angle of the cone is $\varphi$. The beams of the $\mathrm{CW}$ lidar are focused at some distance, $d_{\mathrm{f}}$. If other measurement planes are required, refocusing of the laser beam is necessary. For the pulsed lidar studied here $(N=5)$, the beam directions also form a cone where four positions are within the conical surface and one is perpendicular to the rotor plane (Fig. 1, right panel).

The $i$ th lidar beam points to the direction defined by the unit vector $\boldsymbol{n}_{i}(i=1, \ldots, N)$. The unit vector can be expressed as $\boldsymbol{n}=(-\cos \varphi, \sin \varphi \cos \theta, \sin \varphi \sin \theta)$, where $\theta$ is the angle between the $y$ axis and $\boldsymbol{n}$ projected onto the $y-z$ plane. For the beam perpendicular to the rotor, $\boldsymbol{n}=$ $(-1,0,0)$. If we assume that the lidars measure at a point, instead of over a probe volume, and that the $u, v$, and $w$ components do not change over the scanned area, the radial velocity of the lidar beams over the scanned circle can be estimated as

$v_{\mathrm{r}}(\theta)=-u \cos \varphi+v \sin \varphi \cos \theta+w \sin \varphi \sin \theta$.

\subsection{Lidar radial velocity spectra and beam variances}

Mann et al. (2009) and Sjöholm et al. (2009) show an expression for the spectra measured by a lidar beam

$F_{v_{\mathrm{r}}}\left(k_{1}\right)=n_{i} n_{j} \iint|\hat{\phi}(\boldsymbol{k} \cdot \boldsymbol{n})|^{2} \Phi_{i j}(\boldsymbol{k}) \mathrm{d} k_{2} \mathrm{~d} k_{3}$,

where $\hat{\phi}$ is the Fourier transform of the lidar weighting function that considers the probe volume. For a $\mathrm{CW}$ lidar, this is typically approximated by

$\hat{\phi}\left(k_{1}\right)=\exp \left(-|k| z_{\mathrm{R}}\right)$,

where $z_{\mathrm{R}}$ is the Rayleigh length (Sonnenschein and Horrigan, 1971) that can be estimated as

$z_{\mathrm{R}}=\frac{\lambda d_{\mathrm{f}}^{2}}{\pi r_{\mathrm{b}}^{2}}$

where $\lambda$ is the laser wavelength and $r_{\mathrm{b}}$ the beam radius at the output lens. For the pulsed lidar

$\hat{\phi}\left(k_{1}\right)=\operatorname{sinc}^{2}\left(k z_{\mathrm{R}} / 2\right)$.

In Eq. (9) $z_{\mathrm{R}}$ is not the Rayleigh length as in Eq. (7), but rather half the length of a rectangular pulse (Mann et al.,
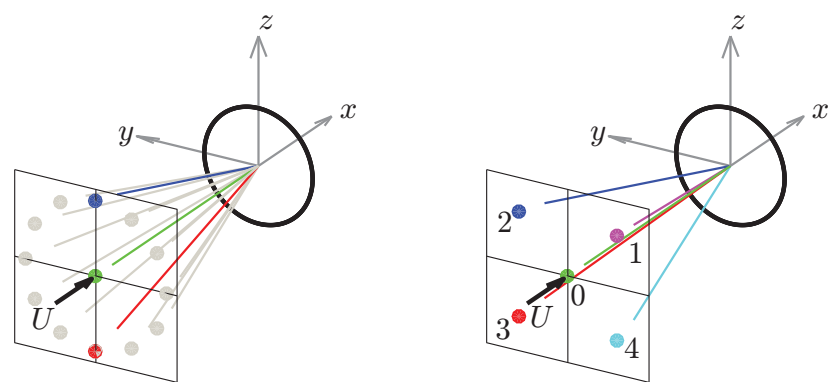

Figure 1. Geometry of the rotor and nacelle lidars. The $x$ axis is in the mean wind direction. The lidar beams point upwind in the directions determined by the unit vectors $\boldsymbol{n}_{i}$. For the CW lidar (left frame) we include a beam perpendicular to the rotor for comparison only. For the pulsed lidar (right frame) we show a five-beam configuration where beam 0 is perpendicular to the rotor. Beams perpendicular to the rotor are shown in green (beam 0), top beams in blue and magenta (beams 1 and 2), bottom beams in red and cyan (beams 3 and 4), and other beams in grey.

2009). Despite this discrepancy, we use the same symbol because $z_{\mathrm{R}}$ is the parameter that characterizes both weighting functions. Notice that $F_{v_{\mathrm{r}}}$ is not a function of $d_{\mathrm{f}} / L$ because the turbulence is assumed homogeneous.

Examples of radial velocity spectra of the $\mathrm{CW}$ and pulsed lidars calculated from Eq. (6) with a half opening angle of $\varphi=15^{\circ}$, which are compared with the "ideal" sonic $u$ spectrum, are shown in Figs. 2 and 3, respectively (within the range of wave numbers that we are interested in, sonic anemometers resolve the $u$ spectrum well). As explained in Mann et al. (2010), the negative correlation between the vertical and horizontal velocities causes the variance of the upward (and forward) pointing beam to generally be the highest of all beams, while the variance is generally the lowest for the downward beam. The difference between the downward and upward pointing beam spectra is smaller than the differences between $u, v$, and $w$ spectra and deteriorates with increasing $z_{\mathrm{R}} / L$. This ratio indicates the amount of filtering of eddies due to the probe volume. We can also see that for the pulsed lidar the radial spectra of the top beams ( 1 and 2) are above the sonic $u$ spectrum for $z_{\mathrm{R}} / L=0.25$, which is due to contributions from different components of the spectral tensor. Similar mechanisms can result in a middle beam radial velocity spectrum above the top beam one, particularly for $z_{\mathrm{R}} / L \geq 1$.

Figure 4's left panel shows the behavior of the ratio of the lidar beam radial velocity variance, $\sigma_{v_{\mathrm{r}}}^{2}$, to the variance of the $u$ component, $\sigma_{u}^{2}$, for a number of $z_{\mathrm{R}} / L$ values and for both types of lidars based on the Mann model with $\Gamma=3$. As expected from the results in Figs. 2 and 3, the ratio increases with decreasing $z_{\mathrm{R}} / L$ and for the $0 /$ middle beam of the pulsed/CW lidars, $\sigma_{v_{\mathrm{r}}}^{2} / \sigma_{u}^{2}=1$ at $z_{\mathrm{R}} / L=0$ as no averaging due to probe volume occurs. Furthermore, both lidars' top beams variances can be higher than $\sigma_{u}^{2}$ for $z_{\mathrm{R}} / L \approx 0$. An- 

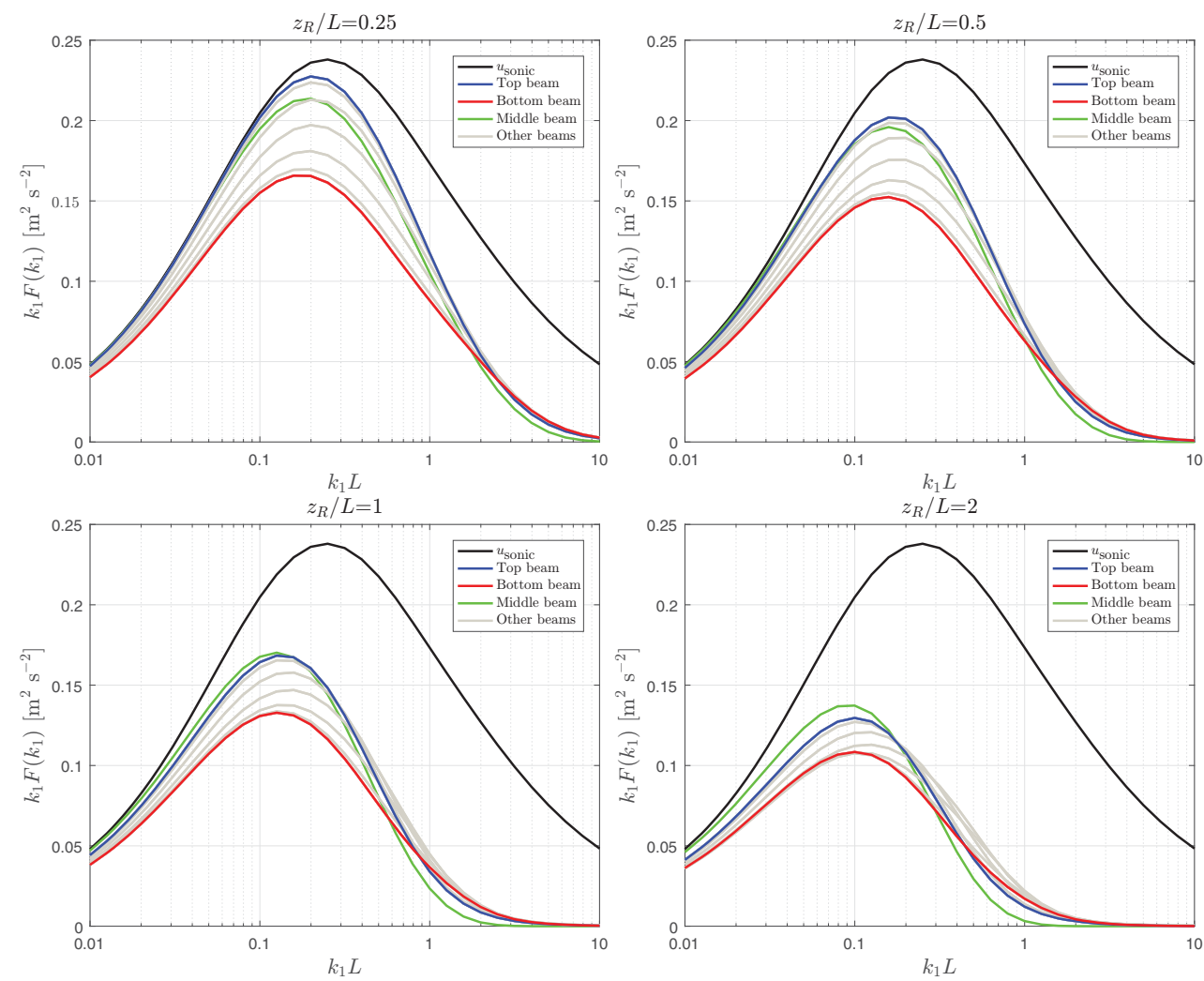

Figure 2. Sonic and CW lidar velocity spectra from Eqs. (3) and (6), corresponding to the beams shown in Fig. 1 (left panel). Values of $z_{\mathrm{R}} / L$ are indicated, $\varphi=15^{\circ}, \Gamma=3$, and $\alpha \varepsilon^{2 / 3}=0.1 \mathrm{~m}^{4 / 3} \mathrm{~s}^{-2}$.

other way to study the contributions of the different velocity components to $\sigma_{v_{\mathrm{r}}}^{2}$ is shown in Fig. 4 right panel. There we illustrate the ratio of the variance of the other two components, $\sigma_{v}^{2}$ and $\sigma_{w}^{2}$, as well as $\sigma_{u}^{2}$ to $\sigma_{v_{\mathrm{r}}}^{2}$ as a function of the beam azimuthal position for $z_{\mathrm{R}} / L=0$. With $\varphi=15^{\circ}$ and such turbulence characteristics, we can only measure a portion of $\sigma_{v}^{2}$ and $\sigma_{w}^{2}$, and $\sigma_{v_{\mathrm{r}}}^{2} \approx \sigma_{u}^{2}$ at $\theta \approx 11^{\circ} / 169^{\circ}$ (also if a middle beam is used, no matter the turbulence characteristics). For the same turbulence characteristics as those used in Fig. 4, if we use a lidar with $\varphi=60^{\circ}, \sigma_{v_{\mathrm{r}}}^{2}<\sigma_{u}^{2}$ for all azimuthal positions, whereas $\sigma_{v_{\mathrm{r}}}^{2} \approx \sigma_{v}^{2}$ at $\theta \approx 200^{\circ} / 340^{\circ}$ and $\sigma_{v_{\mathrm{r}}}^{2} \approx \sigma_{w}^{2}$ at $\theta \approx 237^{\circ} / 303^{\circ}$ (not shown). It is also observed that for the same $z_{\mathrm{R}} / L$ value, the averaging by the $\mathrm{CW}$ lidar has a stronger effect on the variance than the pulsed lidar.

\section{Unfiltered lidar radial velocity variance}

The unfiltered variance of the lidar beams, $\sigma_{v_{r} \text { unf }}^{2}$, can be estimated by using the information of the instantaneous Doppler radial velocity spectrum. Following the steps in Mann et al. (2010) or Branlard et al. (2013), the ensembleaverage Doppler spectrum of the radial velocity $\left\langle S\left(v_{\mathrm{r}}\right)\right\rangle$ can be assumed to be equal to the probability density function of $v_{\mathrm{r}}$, i.e., $\left\langle S\left(v_{\mathrm{r}}\right)\right\rangle=p\left(v_{\mathrm{r}}\right)$. This is because the average of $v_{\mathrm{r}}$ along the beam does not change highly with radial distance, as FL nacelle lidars use a small cone angle and so the velocity gradient along the probe volume is negligible. Therefore, $\sigma_{v_{r, \text { unf }}}^{2}$ can be estimated as the second central moment of $p\left(v_{\mathrm{r}}\right)$.

Assuming homogeneous turbulence, once $\sigma_{v_{r} \text { unf }}^{2}$ is computed, the scanning pattern can be used to extract the velocity-component variances by taking the variance of $v_{\mathrm{r}}$ in Eq. (5),

$$
\begin{aligned}
\sigma_{v_{r, \text { unf }}}^{2}(\theta) & =\sigma_{u}^{2} \cos ^{2} \varphi+\sigma_{v}^{2} \sin ^{2} \varphi \cos ^{2} \theta+\sigma_{w}^{2} \sin ^{2} \varphi \sin ^{2} \theta \\
& -2 \overline{u^{\prime} w^{\prime}} \cos \varphi \sin \varphi \sin \theta,
\end{aligned}
$$

where $\overline{u^{\prime} w^{\prime}}$ is the $u w$ covariance, the primes denote fluctuations, and the overbar a time average, and we ignore the terms where $\overline{u^{\prime} v^{\prime}}$ and $\overline{v^{\prime} w^{\prime}}$ appear because these two are usually small ( $u$ fluctuations are in the mean wind direction). In the case of misalignment of the lidar beams with respect to the wind, because of either misalignment of the turbine with the wind (yaw misalignment), lidar misalignment with the turbine, or both, it is not difficult to derive an expression for $\sigma_{v_{r, \text { unf }}}^{2}$ that accounts for the misalignment angle $\beta$. 

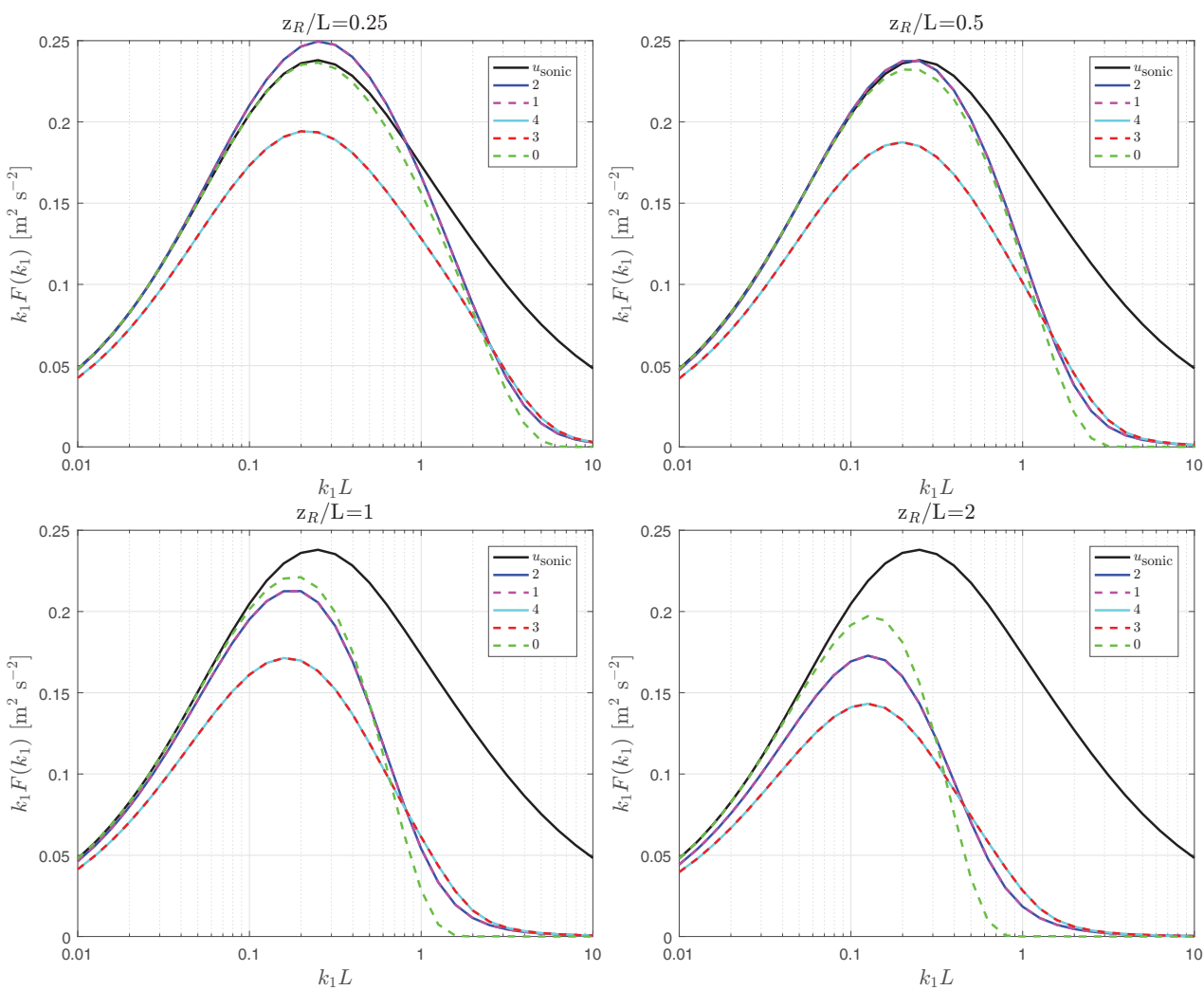

Figure 3. Sonic and pulsed lidar velocity spectra from Eqs. (3) and (6) corresponding to the beams shown in Fig. 1 (right panel). Values of $z_{\mathrm{R}} / L$ are indicated, $\varphi=15^{\circ}, \Gamma=3$, and $\alpha \varepsilon^{2 / 3}=0.1 \mathrm{~m}^{4 / 3} \mathrm{~s}^{-2}$.

\subsection{Turbulence characterization from nacelle-lidar measurements}

The strategy is to calculate theoretical spectra (in the form of a LUT) that include both the effect of pointing the lidar in the direction $\boldsymbol{n}_{i}$ and of averaging. Then, the measured spectra are fitted to the LUT to get the turbulence parameters. One can expect this procedure to be unsuccessful for $z_{\mathrm{R}}>L$, i.e., if the lidar is averaging out most eddies as shown in Figs. 2 and 3 .

The computational burden of creating a lidar-based LUT using Eq. (6) is larger than in the standard case, i.e., using Eq. (4), because $F_{v_{\mathrm{r}}}$ is not only a function of the two parameters $k_{1} L$ and $\Gamma$ but also of $z_{\mathrm{R}} / L, \varphi$, and $\theta$. Furthermore, lidar beam misalignment can be an issue. Therefore, we need to add an extra dimension to the LUT because such misalignment has a large effect on the lidar radial velocity spectrum.

Figure 5 illustrates the effect of misalignment $\left(\beta=-2^{\circ}\right)$ on the pulsed lidar radial velocity spectra for a set of Mann parameters. The effect of the relatively small misalignment is noticeable; the spectrum of the beams that become more parallel to the wind is clearly above that of those that become less parallel at the same height. For this particular pulsed lidar configuration, misalignment can result in a similar spec- trum for the beams 0 (middle) and $1 / 2$ (depending on the sign of the misalignment).

\section{Site and measurements}

\subsection{Site}

The Nørrekær Enge wind farm is located in the Himmerland region in northern Jutland, Denmark, $\approx 300-400 \mathrm{~m}$ southeast of the waters of Limfjorden (see Fig. 6). It comprises 13 Siemens 2.3 MW-93 wind turbines with hub height of $81.8 \mathrm{~m}$ and a rotor diameter $D$ of $92.6 \mathrm{~m}$. They are aligned on a row at a direction $73.9^{\circ}$ with the north. The distance between the turbines is $487 \mathrm{~m}(5.2 \mathrm{D})$. A meteorological mast was located at $101.2^{\circ}$, at a distance of $232 \mathrm{~m}(2.5 \mathrm{D})$ from turbine number 4 (from left to right on the row). The wind farm is located over flat terrain and the surface is characterized by a mix between croplands and grasslands, and the fjord to the north. At $\approx 2 \mathrm{~km}$ southwest of turbine 4 , the terrain is no longer flat.

\subsection{Measurements}

The measurements here analyzed correspond to the period 27 October 2015 to 7 January 2016. There are three types of measurements: supervisory control and data acquisition 

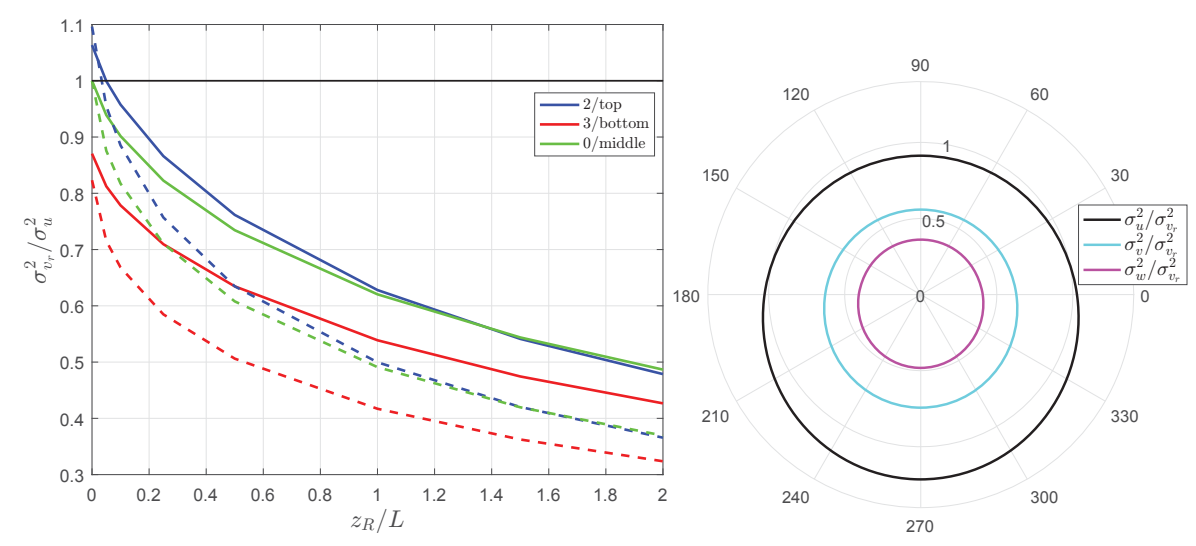

Figure 4. (Left) Variance of the lidar beams' radial velocity (divided by the $u$ component variance) of the $2 /$ top, 3/bottom and 0/middle beams of the pulsed/CW (solid/dashed lines) lidars as a function of $z_{\mathrm{R}} / L$. (Right) Ratio of the variance of each of the velocity components to that of lidar beam as function of azimuthal position for $z_{\mathrm{R}} / L=0$. Turbulence characteristics are computed for $\varphi=15^{\circ}$ and $\Gamma=3$.

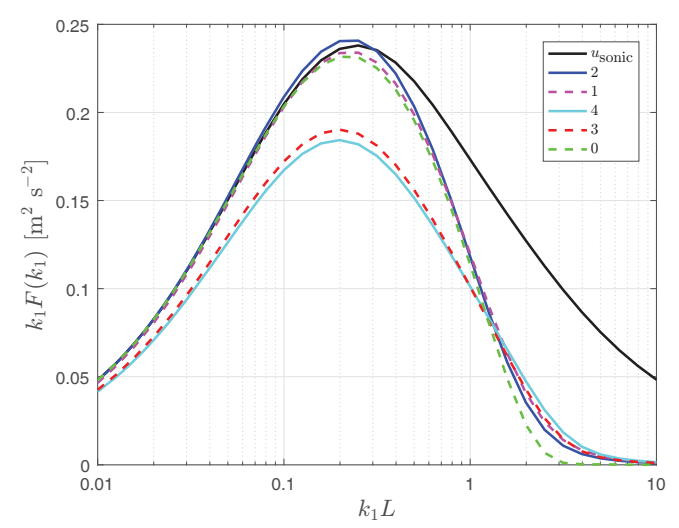

Figure 5. Effect of lidar beam misalignment (with respect to the wind) on the radial velocity spectra of a pulsed lidar for $\varphi=15^{\circ}$, $\Gamma=3, \alpha \varepsilon^{2 / 3}=0.1 \mathrm{~m}^{4 / 3} \mathrm{~s}^{-2}, z_{\mathrm{R}} / L=0.5$, and $\beta=-2^{\circ}$.

(SCADA) on turbine 4, FL nacelle-lidar measurements from systems mounted on the nacelle of turbine 4, and meteorological mast observations. Both lidars were pre-tilted down $\approx 0.30^{\circ}$ so that their axes pointed at hub height, at a position $2.5 \mathrm{D}$ from the turbine for maximum power-performance operating conditions, based on aeroelastic simulations of the tower bending (A. Vignaroli, personal communication, 2016).

\subsubsection{Turbine measurements}

For this analysis we use the following SCADA 10 min means of turbine 4: yaw, power, and turbine and grid status. The yaw and power signals provide measurements of the position of the turbine and the converted power, and the grid and turbine status signals show whether the turbine was grid-connected (yes/no) and available (yes/no).

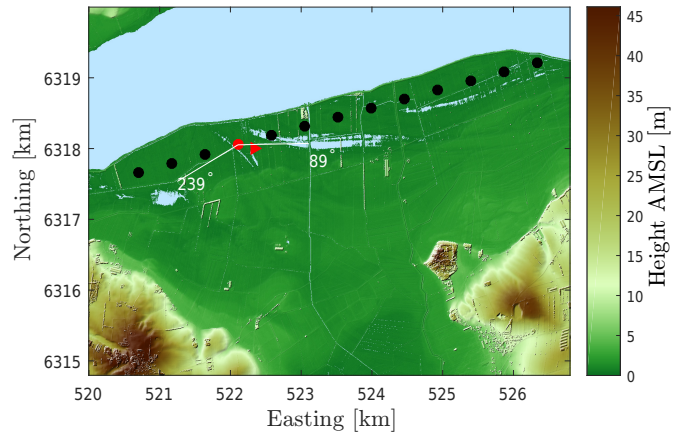

Figure 6. The Nørrekær Enge wind farm in northern Denmark on a digital surface elevation model (UTM32 WGS84). The wind turbines are shown in circles, that with the nacelle lidars in red and the mast in a triangle. The sector used for the analysis is also indicated. The waters of Limfjorden are shown in light blue.

\subsubsection{Pulsed lidar}

A five-beam Avent pulsed lidar (hereafter known as Avent) was mounted on the nacelle of turbine 4 . Ten different ranges were measured simultaneously per beam position $(49,72,95$, $109,121,142,165,188,235$, and $281 \mathrm{~m}$ ). The beam configuration is exactly as that in the right panel of Fig. 1 (and we will use the same beam numbering), with $\varphi=15.08^{\circ}$ and $z_{\mathrm{R}}=24.75 \mathrm{~m}$ (Borraccino et al., 2015). The lidar accumulated radial velocity spectra per beam position for $1 \mathrm{~s}$ before it moved to the next beam position; thus, radial velocity time series can be analyzed at $0.2 \mathrm{~Hz}$. Each radial velocity estimate from the average Doppler spectrum was performed by the instrument using a maximum-likelihood-estimator algorithm (Peña et al., 2015). 


\subsubsection{Continuous-wave lidar}

A ZephIR dual-mode CW lidar (hereafter known as ZephIR) was also mounted on the nacelle of turbine 4 . Five different ranges were considered $(10,30,95,120$, and $235 \mathrm{~m})$; for each range $\approx 50$ azimuthal positions on the circle formed with a cone with $\varphi=15.05^{\circ}$ were measured during $1 \mathrm{~s}$; the system averaged Doppler radial velocity spectra within azimuthal ranges of $\approx 7.38^{\circ}$ to get an estimate of the radial velocity per azimuth by computing the centroid of the average Doppler spectrum (Borraccino et al., 2015). The system also kept a record of each average Doppler radial velocity spectrum, which is used here to estimate the unfiltered variance. The lidar characteristics $\lambda=1.56 \times 10^{-6} \mathrm{~m}$ and $r_{\mathrm{b}}=28 \mathrm{~mm}$ (M. Harris, personal communication, 2016) can be used to estimate $z_{\mathrm{R}}$ with Eq. (8). Each range was sampled three times before focusing to the next one; thus, radial velocities for the same range and azimuthal position can be found every $\approx 18 \mathrm{~s}$

\subsubsection{Mast measurements}

We use measurements from cup anemometers (P2546A) at 80,78 , and $57 \mathrm{~m}$ height, mounted on $3 \mathrm{~m}$ long booms $250^{\circ}$ from the north; from a 3-D sonic anemometer (CSAT3) at $76 \mathrm{~m}$ on a $2 \mathrm{~m}$ boom $190^{\circ}$ from the north; and a wind vane (Vector W200P) at $78 \mathrm{~m}$ on a $3 \mathrm{~m}$ boom $70^{\circ}$ from the north, all mounted on the meteorological mast. The mast is an equilateral triangular lattice structure with a width of $0.4 \mathrm{~m}$ at $80 \mathrm{~m}$.

\section{Data analysis}

\subsection{Data selection and filtering}

We analyze the time series of all data and their statistics in 10 min periods. The total number of $10 \mathrm{~min}$ periods available for analysis is 9586. The next steps are followed in the analysis:

1. We use the $10 \mathrm{~min}$ vane measurements to concentrate the analysis on a wake-free sector covering the mast location $\left(88.85-238.85^{\circ}\right)$ that takes into account the obliquity of the wind farm row and a $15^{\circ}$ wake expansion (see Fig. 6). A total of $582510 \mathrm{~min}$ measurements are available for analysis where both lidars are also working (based on a 10 min status signal of both lidars) and turbine 4 is grid-connected and available.

2. The availability of the Avent data is highest at the range $121 \mathrm{~m}$ because this range is the closest to the focusing distance. Therefore, we focus all our lidar-data analysis at this range, although the mast is at $232 \mathrm{~m}$ from turbine 4. Furthermore, when a carrier-to-noise (CNR) filter is applied to the $5 \mathrm{~s}$ time series, the two lowest beams ( 3 and 4) return fewer data than the others due to, among other things, obstruction from the blades (the availability of beam 3 is lower than that of beam 4). A total of 323610 min periods are available for analysis after filtering the $5 \mathrm{~s}$ Avent data so that for each $10 \mathrm{~min}$ period there are a minimum of 110 samples for beams $0,1,2$, and 4 with $\mathrm{CNR}>-22 \mathrm{~dB}$.

3. We then extract all radial velocities for all azimuthal positions of the ZephIR for the range $120 \mathrm{~m}$ when no rain was detected by the instrument. The azimuthal position of the $\approx 50$ points over the scanned circle changes after each revolution. A total of $259010 \mathrm{~min}$ periods are available for analysis in which there are a minimum of 4500 radial velocities samples per $10 \mathrm{~min}$ period at the $120 \mathrm{~m}$ range.

4. Finally, we extract the $1 \mathrm{~Hz}$ data of the sonic anemometer and cup anemometer at $80 \mathrm{~m}$, in which there are a minimum of 600 samples per $10 \mathrm{~min}$ period. The final dataset thus contains 227310 min samples of concurrent turbine-lidars-mast data.

Furthermore, each $10 \mathrm{~min}$ time series has been postprocessed. For the Avent data, we linearly detrend each radial velocity time series for each beam before applying a despiking filter, where values above and below 3 standard deviations from the mean are filtered out. The missing values are then filled in using linear interpolation. The top left panel of Fig. 7 shows an example of a 10 min time series of the Avent beams' radial velocity. The solid lines show the final interpolated time series and the markers show original radial velocities before post-processing.

For the ZephIR data, we construct time series of radial velocities at azimuthal positions similar to those of the Avent. Since the azimuthal positions of the ZephIR change from revolution to revolution, we extract radial velocities within azimuthal position bins of $7.2^{\circ}$ on a fixed frame of reference. Three of such bins, 43, 6, and 31, are "aligned" with the Avent beams 1,2, and 4, respectively. The time series per bin is then threshold-filtered with a minimum radial velocity of $2 \mathrm{~m} \mathrm{~s}^{-1}$, and detrended and despiked as with the Avent data. The top right panel of Fig. 7 shows the time series per bin; we include four more bins $(0,12,18$, and 37) than those aligned with the Avent beams and their positions can be inferred by color coding using the bottom left panel of Fig. 7 which shows the radial velocities in a polar plot. In the top panels in Fig. 7 the effect of despiking is noticeable (the filtered time series are shown in solid lines and the original are shown in markers), and in the bottom left panel of Fig. 7, all the radial velocities estimated from the Doppler spectrum within the $10 \mathrm{~min}$ period at the $120 \mathrm{~m}$ range by the ZephIR are shown. Since the lidars were mounted behind the rotor, the rotating blades sometimes interfered with the beam and the estimated radial velocity became the projection of the radial velocity of the blade onto the beam direction; the result is the figure of eight close to zero radial velocity shown in 

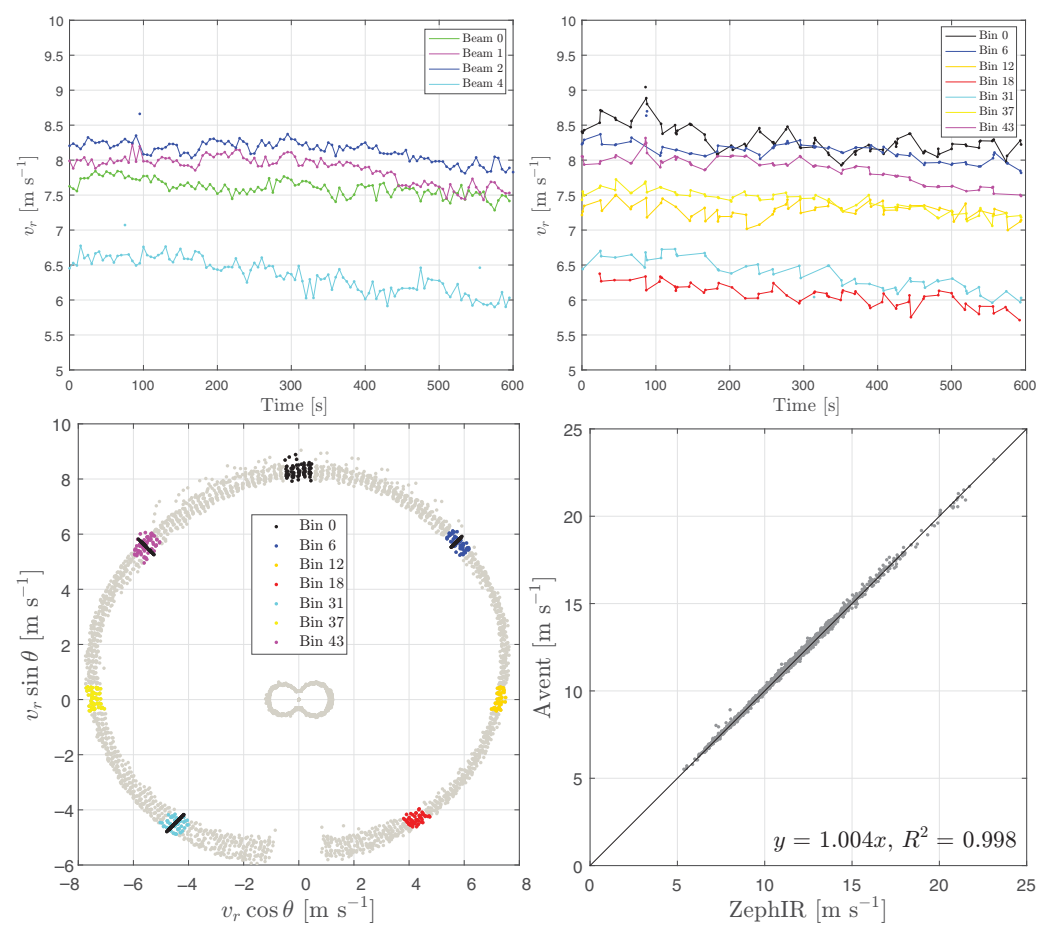

Figure 7. An example of a $10 \mathrm{~min}$ time series of the radial velocity of different beams for the Avent (top left) and the ZephIR (top right) lidar. The radial velocities of the two lidars at all azimuthal positions are illustrated in the bottom-left panel (see text for details). In the bottom-right panel, we show a comparison of the 227310 min mean radial velocities of the Avent (beam 2) and ZephIR (bin 6) with the results of a linear regression through the origin and coefficient of determination $R^{2}$. Measurements from beam 3 of the Avent are omitted due to low availability.

the bottom left panel of Fig. 7. In this latter plot, we also include the radial velocities of the three Avent beams that are aligned with the ZephIR bin positions. At these three positions, both lidars show good agreement; a comparison of all 10 min mean radial velocities estimated by the Avent and ZephIR for one of these "aligned" positions, beam 2 and bin 6 , respectively, is shown in the bottom right panel of Fig. 7. The top right panel of Fig. 7 also shows that it is possible to get more than one radial velocity value within the same azimuthal bin (sometimes up to three values). Finally, the ZephIR's time series are "completed" using linear interpolation.

For each $10 \mathrm{~min}$ period, the $1 \mathrm{~Hz}$ sonic and cup anemometer data are detrended and despiked as with the lidar data, and mean and turbulence statistics are computed. The sonicanemometer wind-speed components are rotated so that $u$ is aligned with the mean wind. We estimate the friction velocity, from the sonic wind speed and temperature fluctuations, as

$u_{*}=\left({\overline{u^{\prime} w^{\prime}}}^{2}+{\overline{v^{\prime} w^{\prime}}}^{2}\right)^{1 / 4}$

and the Obukhov length estimated as

$L_{\mathrm{O}}=-\frac{u_{*}{ }^{3}}{\kappa(g / \bar{T}) \overline{w^{\prime} \Theta_{\mathrm{v}}^{\prime}}}$ where $\kappa$ is the von Kármán constant $(\approx 0.4), g$ the gravitational acceleration, $T$ a reference temperature, and $\Theta_{\mathrm{v}}$ the virtual potential temperature. Spectra of all lidar radial velocities, sonic-anemometer wind speed components and cupanemometer horizontal wind velocity are computed for each $10 \mathrm{~min}$ period. All $10 \mathrm{~min}$ turbulence statistics and spectra from the sonic anemometer are also computed on a 5 and a $18 \mathrm{~s}$ basis, mimicking the lidar sampling frequencies.

\subsection{Sonic-anemometer measurements}

When compared to the measurements from the $80 \mathrm{~m}$ cup anemometer, the sonic-anemometer mean horizontal wind speeds are $2.6 \%$ lower (see Fig. 8 left panel). This bias is higher than $0.6 \%$, which is the estimation that results from assuming, between the two instruments' heights, the logarithmic wind profile

$U=\frac{u_{*}}{\kappa} \ln \left(\frac{z}{z_{0}}\right)$,

where $z_{0}$ is the roughness length, $\approx 0.012 \mathrm{~m}$, which is a typical value of these surface conditions (Peña et al., 2016). When looking at variances, the bias is $12 \%$ (Fig. 8, right panel) if we use the $u$ component or the combined $u$ and $v$ components for the estimation of the sonic-anemometer 

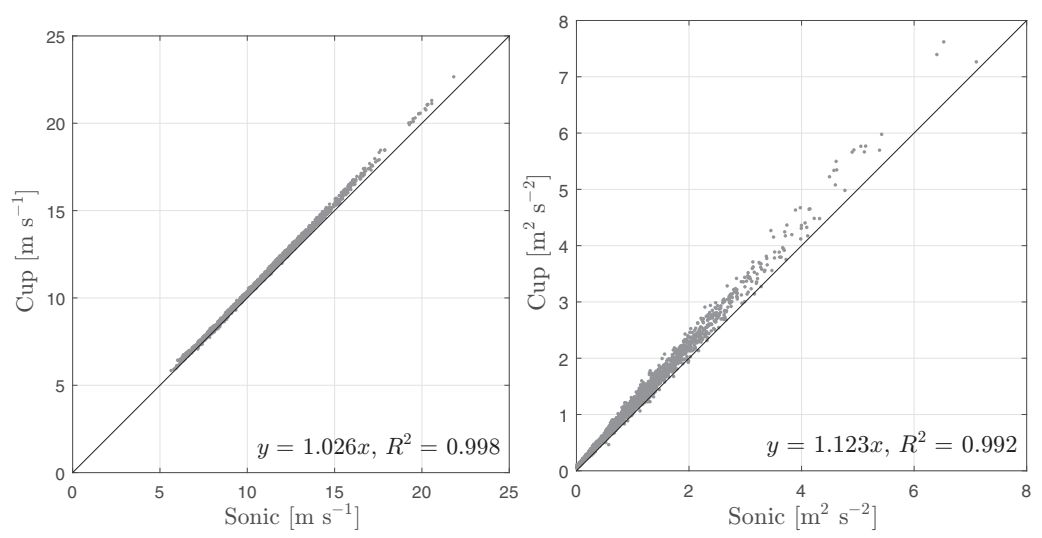

Figure 8. Comparison of sonic and $80 \mathrm{~m}$ cup anemometer statistics: mean wind speed (left frame) and horizontal-wind variance (right frame). Each 10 min sample is shown in grey markers, a 1:1 line is shown for guidance in black, and the results of a linear regression through the origin and $R^{2}$ are given.

variance. The latter means that for this site and at this height, the $v$ variance has a low contribution to the horizontal velocity variance (which is what a cup anemometer does theoretically measure) and so we could assume the cup-anemometer variance to give a good estimate of the $u$ variance. On the other hand, the bias between both instruments' variances cannot be explained simply; a $4 \%$ bias is expected, assuming the $2 \%$ bias of the mean wind speed.

The behavior of the sonic-derived velocity spectra does not correspond well with the notion of turbulence local isotropy within the inertial subrange, where we expect the same spectral density for the $v$ and $w$ components and the $u$ component is $25 \%$ lower (Wyngaard, 2010). Figure 9 shows that within the inertial subrange the ensemble-average sonic $u$ spectrum (of all 10 min observed spectra) is indeed $\approx 25 \%$ lower than the $v$ spectrum but so is the $w$ spectrum. Possible explanations for this are path-averaging errors and transducer shadowing mainly attenuating the $w$ spectrum measured by the CSAT3 (Horst and Oncley, 2006). Figure 9 also illustrates the fit to the three auto-spectra and cross-spectrum using the Mann model (see Sect. 2), which shows the expected behavior within the inertial subrange $\left(k_{1} \gtrsim 0.03 \mathrm{~m}^{-1}\right.$ for this case). The fit is performed on the ensemble-average spectra that have been logarithmically-averaged on the basis of the wavenumber (we will use such logarithmicallyaveraged spectra when fitting Mann parameters). These "average" Mann parameters $\left(\Gamma=3.00, \alpha \varepsilon^{2 / 3}=0.14 \mathrm{~m}^{4 / 3} \mathrm{~s}^{-2}\right.$, and $L=35.38 \mathrm{~m}$ ) are similar to those observed at a site with similar surface and turbulence characteristics (Peña et al., 2010a), but it should be noticed that these are the average of spectra for a number of atmospheric and turbulence conditions and that the Mann-model fitting procedure is normally performed over specific wind speed, turbulence, or atmospheric-stability ranges.

Due to the uncertainty on the sonic-derived statistics, we will use the cup-anemometer variance as a proxy for $\sigma_{u}^{2}$.

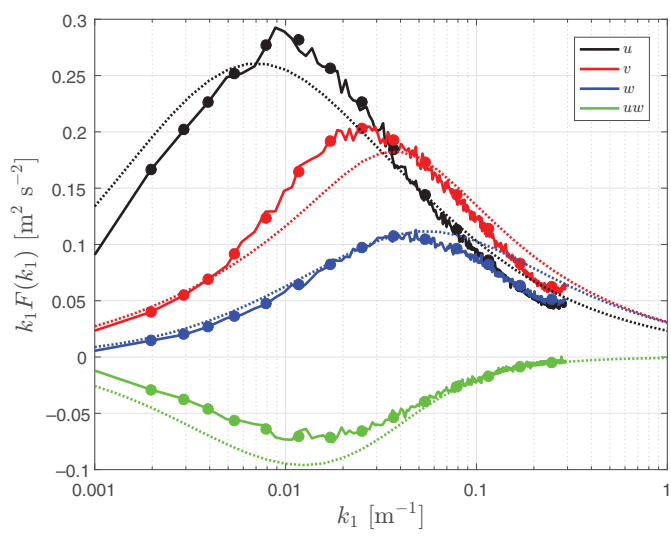

Figure 9. Power spectrum for different velocity components. The solid lines show the ensemble-average spectra of all $10 \mathrm{~min}$ sonicanemometer spectra; the markers, the $k$ based logarithmicallyaverage spectra of all $10 \mathrm{~min}$ spectra; and the dotted lines, a fit to the spectra using the Mann model.

However, we will use the sonic-based Mann parameters for comparison with the lidar-based Mann parameters (and for estimations of $\sigma_{v, w}^{2}$ and $\overline{u^{\prime} w^{\prime}}$ ) because it is the only reference we have for three-dimensional turbulence measurements.

\subsection{Undersampling and noise removal}

Although the variances of a velocity time series sampled over a $10 \mathrm{~min}$ period at a frequency $f_{s}$ of 0.2 or $0.06 \mathrm{~Hz}$ are not statistically different from those estimated from 1 or $10 \mathrm{~Hz}$ records, aliasing and noise might appear both in the sonicanemometer and the lidar radial velocity spectra. The left panel of Fig. 10 shows the Avent radial velocity spectrum that has been ensemble-averaged from all the $10 \mathrm{~min}$ observed spectra for each of the beams. We conjecture that the increase in the spectral densities at high frequencies is due to noise. 

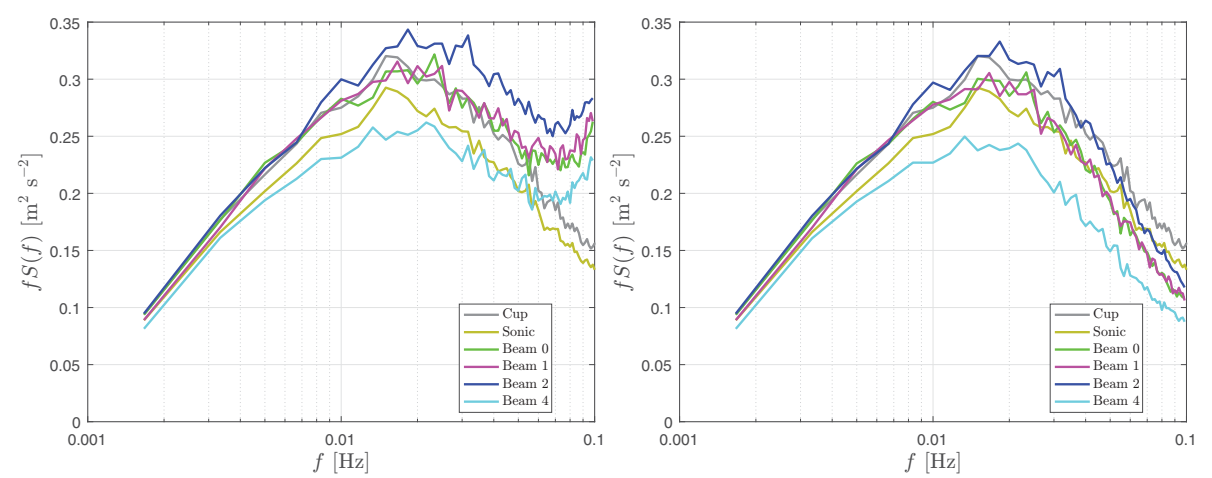

Figure 10. Ensemble-average spectrum of all $10 \mathrm{~min}$ Avent radial velocity spectra (per beam), sonic-anemometer $u$ spectrum, and $80 \mathrm{~m}$ cup-anemometer spectrum. Original (left) and noise-filtered lidar radial velocity spectra (right).

The right panel of Fig. 10 shows the effect of a noise filter, which is based on the method by Kirchner (2005), on the ensemble-average Avent radial velocity spectra.

The noise filter seems to recover the shape of the Avent radial velocity spectra. However, when tested on the $18 \mathrm{~s}$ sonic ensemble-average $u$ spectrum (not shown), the filter highly distorts the shape and the peak of the spectrum. Therefore, we focus the spectra analysis on the measurements performed at $f_{s} \geq 0.2 \mathrm{~Hz}$, i.e., we exclude the ZephIR radial velocity spectra for the analysis. For the results presented hereafter, the noise filter is only applied to the Avent radial velocity spectra.

Figure 10 also shows that for these ensemble-averages, the spectral density of beam 2 is the highest, followed by that of beams 0 and 1 , and then that of beam 4 . This behavior might be due to three reasons: Excessive rolling of the Avent, so that beam 2 points higher than beam 1; that the turbulence characteristics at the position of beam 2 are rather different than those at the position of beam 1; or that there is yaw misalignment so that beam 2 points closer to the direction of the mean wind compared to beam 1 (see Fig. 5). Both ZephIR and Avent have tilt and roll signals, and for the $10 \mathrm{~min}$ samples analyzed here the maximum absolute 10 min mean tilt and roll are only 0.56 and $0.31^{\circ}$, respectively. Also, the very flat terrain characteristics should not have such an impact on the ensemble-average spectrum of two beams that point at the same height, like beams 1 and 2 in this particular case. So, the most plausible explanation is that beams 2 and 3 are more aligned with the mean wind than beams 1 and 4 .

In the right panel of Fig. 10, we can see that the spectrum of beam 2 is slightly higher than that of the $80 \mathrm{~m}$ cup anemometer and higher than that of the sonic anemometer (up to $f \approx 0.04$ and $0.07 \mathrm{~Hz}$, respectively). Such behavior is expected for low $z_{\mathrm{R}} / L$ values (see Fig. 3 top left panel) or under lidar misalignment conditions (see Fig. 5). We can also see that the cup-anemometer spectrum is higher than that of the sonic anemometer, as expected from the variance results in the right panel in Fig. 8.

\subsection{Horizontal wind-speed reconstruction}

For both lidars we need to reconstruct the horizontal wind speed at the specific range of the lidars, which can later be used for spectral analysis and for filtered along-wind variance estimates. We use a simplified version of the lineargradient model of Hardesty et al. (1981),

$v_{\mathrm{r}}(\theta)=-\cos \varphi\left(u+R_{\mathrm{d}} \frac{\mathrm{d} u}{\mathrm{~d} z} \sin \theta\right)+v \cos \theta \sin \varphi$,

where $R_{\mathrm{d}}$ is the radius of the disc formed by the scanning pattern at the given range, to estimate $u, v$, and the vertical gradient of the along-wind component, $\mathrm{d} u / \mathrm{d} z$. In Eq. (14), we ignore $w$ and other vertical and horizontal gradients of the wind components because their contribution is small. For both lidars, the beams selected in Sect. 5.1 are used for the reconstruction, which can be done on the time-series basis or the $10 \mathrm{~min}$ averages. Figure 11 shows the results of the lidarbased reconstruction on all 10 min means compared to the $80 \mathrm{~m}$ cup anemometer and between the lidars; for both lidars we show the horizontal wind-speed magnitude but when using the mean wind speed we obtain the same results. The same results, regarding the linear regression and $R^{2}$ (not shown), as those given in the left panel of Fig. 11 are found when comparing the radial velocity of beam 0 with the $80 \mathrm{~m}$ cup-anemometer wind speed on a $10 \mathrm{~min}$ basis.

\subsection{Ensemble-average Doppler radial velocity spectrum}

The Doppler-spectrum analysis is performed over all the 227310 min periods using the ZephIR data (the Doppler spectrum information is not available for the Avent). While each of the $10 \mathrm{~min}$ radial velocity time series per bin position is thresholded and despiked (see Sect. 5.1), we extract the normalized Doppler radial velocity spectrum for each of the samples within that $10 \mathrm{~min}$ and bin position. We then sum all the normalized Doppler spectra within the $10 \mathrm{~min}$ period and the resulting Doppler spectrum is normalized to unit area before we estimate the variance in two ways: by 

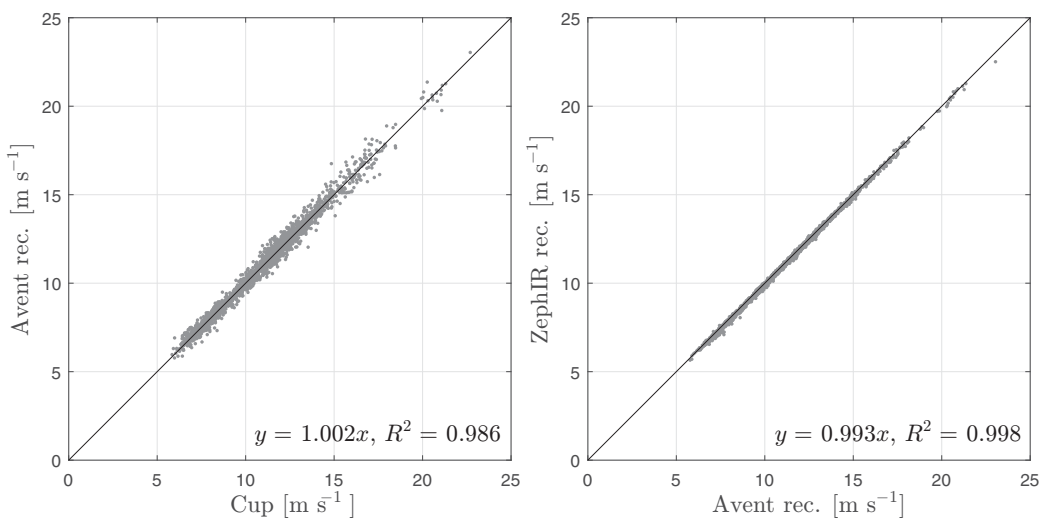

Figure 11. Comparison of reconstructed and $80 \mathrm{~m}$ cup anemometer horizontal wind speeds. (Left) Cup anemometer against Avent. (Right) Avent against ZephIR. Each 10 min sample is shown in grey markers. A $1: 1$ line is shown for guidance in black, and the results of a linear regression through the origin and $R^{2}$ are also given.

computing the second moment from the spectrum and by fitting a normal distribution to the spectrum to extract its variance (Mann et al., 2010; Branlard et al., 2013). Figure 12 illustrates examples of ensemble-average Doppler spectra for different $10 \mathrm{~min}$ periods for the positions of bins 0 and 31 , where we intentionally show $10 \mathrm{~min}$ radial velocity distributions with high and low mean values, and high and low variances, including double-peak distributions (there are only a few of them). These few distributions give us an idea of the variety of turbulence characteristics of the dataset. Distributions with high and low radial velocities generally show high and low variances, respectively, as expected. Particularly in the examples, there is a 10 min period with very low variance for both bin positions with clear larger radial velocities for bin 0 compared to those for bin 31, indicating very high wind shear, which is normally associated with atmospheric stable conditions. This is an early morning $10 \mathrm{~min}$ period in late October, in which the sonic-derived $L_{\mathrm{O}}$ value is $1.82 \mathrm{~m}$, corresponding to extremely stable conditions.

\section{Results}

The results are divided into five parts. In Sect. 6.1, we illustrate the main turbulence characteristics of the site, which we use to classify the data in a number of atmospheric-stability and wind-speed ranges. In Sect. 6.2, we intercompare the ZephIR estimates of variances and co-variances using the unfiltered lidar radial velocity variances with the cup- and sonic-anemometer estimates. Section 6.3 shows the effect of the noise filter on the Avent radial velocity variance for the atmospheric-stability and wind-speed ranges. In Sect. 6.4, we explore the effect of atmospheric stability on both the sonic and the lidar radial turbulence spectra and intercompare the Mann parameters derived from both types of spectra. Finally, in Sect. 6.5, we perform the same exercise as in Sect. 6.4 but on the basis of the wind-speed ranges.

\subsection{Turbulence characteristics}

Figure 13 shows the overall turbulence characteristics of the site based on cup- and sonic-anemometer observations, using the $227310 \mathrm{~min}$ concurrent data. In the left frame, we illustrate the behavior of the turbulence intensity, $\sigma_{U} / U$, with wind speed, using the $80 \mathrm{~m}$ cup-anemometer measurements; wind speeds are in the range $\approx 5-23 \mathrm{~m} \mathrm{~s}^{-1}$ with low $\sigma_{U} / U$ values within the low wind-speed range and $\sigma_{U} / U$ increasing with wind speed. In the right frame, we illustrate the behavior of the dimensionless wind shear, $\phi_{m}=$ $\left(\kappa z / u_{*}\right) \partial U / \partial z$, with dimensionless atmospheric stability, $z / L_{\mathrm{O}}$; we use the cup-anemometer wind-speed measurements at 78 and $56 \mathrm{~m}$ to estimate $\partial U / \partial z(\approx \Delta U / \Delta z)$ and the sonic-derived $u_{*}$ to compute $L_{\mathrm{O}}$ and $\phi_{m}$. Figure. 13, in the right panel, shows that the atmosphere during the analyzed period is mostly stable $\left(z / L_{\mathrm{O}}>0\right)$ and that, as expected, $\phi_{m}$ increases with increasing $z / L_{\mathrm{O}}$. Such atmospheric conditions explain the low $\sigma_{U} / U$ values for low wind speeds. In the left panel of Fig. 13, we include a prediction of $\sigma_{U} / U$, using Eq. (13) with $\sigma_{U}=2.5 u_{*}$ and $z_{o}=0.012 \mathrm{~m}$, which fairly agrees with the data for high wind speeds only, as expected. In Fig. 13 (right panel), we include, for comparison only, the prediction $\phi_{m}=1+4.7 z / L_{\mathrm{O}}$ from surface-layer theory (Högström, 1988) that is offset with the data because $\phi_{m}$, and so $z / L_{\mathrm{O}}$, is estimated at a mean height of $z=67 \mathrm{~m}$ with only two wind-speed observations that were $22 \mathrm{~m}$ apart, whereas the turbulence estimates are from the sonic anemometer at $76 \mathrm{~m}$.

Based on the observed turbulence characteristics and knowing that we need to average a number of $10 \mathrm{~min}$ spectra to be able to robustly extract the Mann parameters (Peña et al., 2010a), we classify the concurrent data into 10 classes as illustrated in Table 1, ensuring that there are a close to $10010 \mathrm{~min}$ samples per class as a minimum. From the atmospheric-stability classes, we can see that the data comprise mainly stable conditions, with stability 1 be- 

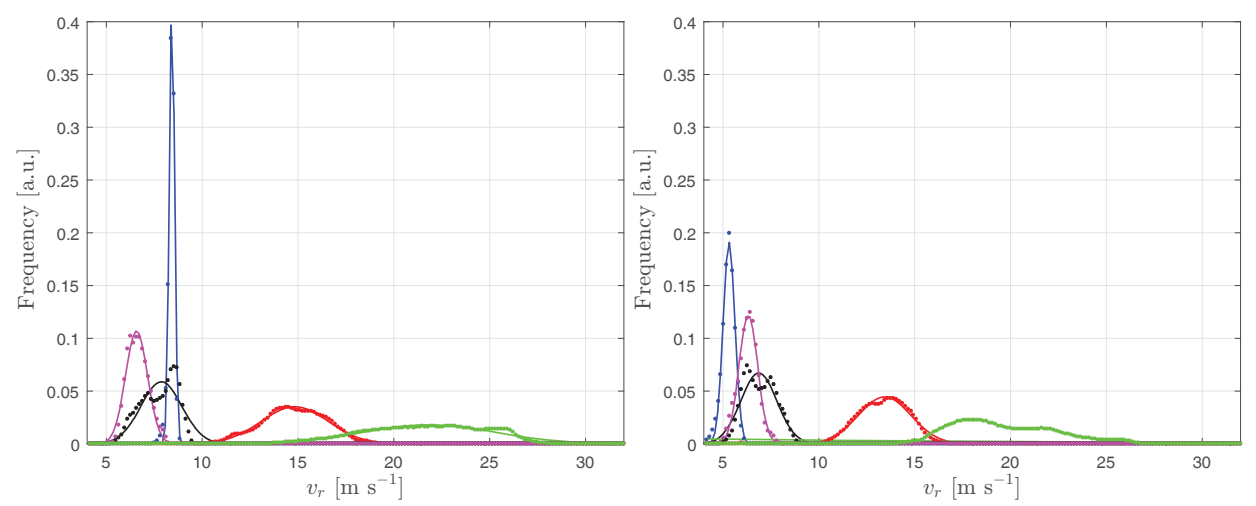

Figure 12. Examples of normalized Doppler radial velocity spectra measured over five 10 min periods with the ZephIR at the positions of bin 0 (left) and bin 31 (right). The markers show the observed distributions and the solid lines show a normal fit.
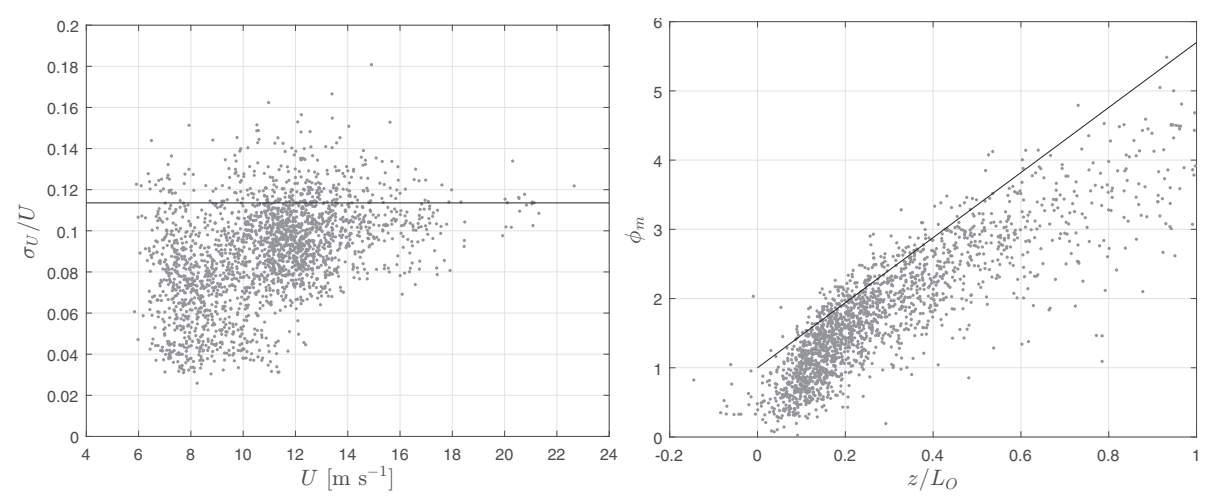

Figure 13. (Left) Turbulence intensity $\sigma_{U} / U$ as a function of mean wind speed $U$ from the 80 m cup-anemometer observations. (Right) Dimensionless wind shear $\phi_{m}$ as a function of dimensionless stability, $z / L_{\mathrm{O}}$, based on the sonic- and cup-anemometer observations. The grey markers show $227310 \mathrm{~min}$ concurrent samples and the solid lines are theoretical predictions (see text for details).

ing the only close-to-neutral class $\left(\left\langle z / L_{\mathrm{O}}\right\rangle=0.0625\right.$, with $z=67 \mathrm{~m})$. The more stable the atmospheric conditions the lower the wind speed and the friction velocity, as expected. Most of the data range within the stability 2 class $\left(\left\langle z / L_{\mathrm{O}}\right\rangle=\right.$ $0.1489)$, i.e., most of the observations are nearly stable. From the wind-speed classes, we observe most of the data within a high-speed range (11-13 $\mathrm{m} \mathrm{s}^{-1}$ ) and, similarly to the stability classification, the lower the wind speed the more stable the atmosphere (except for the speed 1 and 2 classes), and so the lower the friction velocity. Interestingly, for the speed 1 and 2 classes $\widetilde{z / L_{\mathrm{O}}}=0.5084$ and 0.7196 , respectively (where ${ }^{\sim}$ indicates the median value), which are higher values than the mean dimensionless stability of the most stable class (stability 5 ). We use the median for the speed classes since the $L_{\mathrm{O}}$ values highly fluctuate within those speed ranges.

\subsection{Unfiltered lidar turbulence}

Based on the ZephIR configuration $\left(\varphi=15.05^{\circ}\right)$, we are able to predict all variances' ratios $\sigma_{v_{\mathrm{r}}}^{2} / \sigma_{u, v, w}^{2}$, using the Mann model with a given $\Gamma$ parameter for the unfiltered lidar radial velocity variances, i.e., using Eq. (6) with $z_{\mathrm{R}} / L=0$. This is a procedure similar to the one we use for the results in Fig. 4 (right panel). Figure 14 shows a comparison of the ZephIR "unfiltered" radial velocity variances (for bins 0 and 31) with the cup-anemometer variances for all the 227310 min data, together with the Mann-model prediction, using $\Gamma=3$. We present variance estimations that are computed from the normal distribution fit to the average normalized Doppler spectrum, instead of those calculating the second moment from the spectrum, since the latter method is more sensitive to "spurious" data that appear far from the area where most radial velocities are concentrated. This is particularly seen for the lower bins (18 and 31) and might be due to non-filtered blade-obstructed data, noise, or sudden jumps in the radial velocity within the $10 \mathrm{~min}$ period.

As expected, based on the results in Fig. 4, the top (bin 0 ) and a lower beam (bin 31) show a higher and lower variance, respectively, than that of the " $u$ " component (in quotation marks because we use the cup-anemometer measurements). The Mann-model-based results slightly underpredict the ratio $\sigma_{v_{\mathrm{r}}}^{2} / \sigma_{u}^{2}$ for these two beams compared to the raw data. Reducing the value of $\Gamma$ or accounting for misalignment improves the predictions; e.g., with $\Gamma=2.5$ and $\beta=0^{\circ}$ the 
Table 1. Atmospheric-stability and wind-speed classes and ranges based on the cup- and sonic-anemometers' observations (see text for details). The ensemble-average values of the dimensionless stability, wind speed, and friction velocity per range are also provided. For the speed ranges we use the median of the dimensionless stability. $z=67 \mathrm{~m}$ is here the mean height used for the dimensionless atmosphericstability estimates.

\begin{tabular}{lrrrrr}
\hline Class & $z / L_{0}$ & No. of 10 min samples & $\left\langle z / L_{\mathrm{O}}\right\rangle$ & $\langle U\rangle\left(\mathrm{m} \mathrm{s}^{-1}\right)$ & $\left\langle u_{*}\right\rangle\left(\mathrm{m} \mathrm{s}^{-1}\right)$ \\
\hline stability 1 & $-0.1-0.1$ & 225 & 0.0625 & 12.75 & 0.68 \\
stability 2 & $0.1-0.2$ & 629 & 0.1489 & 12.54 & 0.61 \\
stability 3 & $0.2-0.3$ & 350 & 0.2435 & 11.34 & 0.48 \\
stability 4 & $0.3-0.4$ & 225 & 0.3475 & 10.71 & 0.42 \\
stability 5 & $0.4-0.5$ & 153 & 0.4457 & 10.02 & 0.35 \\
\hline class & $U\left(\mathrm{~m} \mathrm{~s}^{-1}\right)$ & no. of 10 min samples & $\langle U\rangle\left(\mathrm{m} \mathrm{s}^{-1}\right)$ & $\widetilde{z / L_{\mathrm{O}}}$ & $\left\langle u_{*}\right\rangle\left(\mathrm{m} \mathrm{s}^{-1}\right)$ \\
\hline speed 1 & $5-7$ & 93 & 6.65 & 0.5084 & 0.21 \\
speed 2 & $7-9$ & 516 & 7.98 & 0.7196 & 0.23 \\
speed 3 & $9-11$ & 506 & 10.07 & 0.3684 & 0.37 \\
speed 4 & $11-13$ & 741 & 11.94 & 0.2133 & 0.52 \\
speed 5 & $13-15$ & 278 & 13.82 & 0.1402 & 0.64 \\
\hline
\end{tabular}
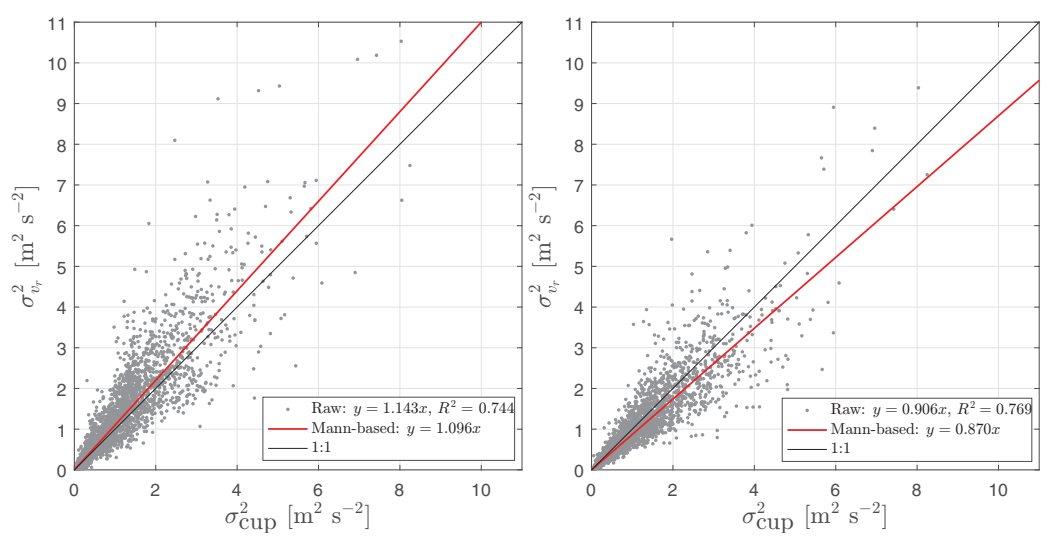

Figure 14. Comparison of the $80 \mathrm{~m}$ cup-anemometer and the unfiltered ZephIR radial velocity variances for bins 0 (left) and 31 (right). We show a $1: 1$ line for guidance and the predictions of the Mann model using $\Gamma=3$. Results of a linear regression through the origin and $R^{2}$ are also given. For bin $31, \sigma_{v_{\mathrm{r}}}^{2}>11 \mathrm{~m}^{2} \mathrm{~s}^{-2}$ for two $10 \mathrm{~min}$ periods.

Mann-model results predict biases of 11 and $-13 \%$ for bins 0 and 31, respectively (not shown). It is important to mention that the original (filtered) radial velocity variances for these two bins are 13 and $31 \%$ lower than the cup-anemometer measurements (not shown) with slightly higher $R^{2}$ values, 0.785 and 0.798 , respectively.

Furthermore, we can also estimate $\sigma_{u, v, w}^{2}$ and $\overline{u^{\prime} w^{\prime}}$ for each $10 \mathrm{~min}$ period through a least-squares fit of Eq. (10) that does not depend on the Mann parameters but assumes homogeneous turbulence within the scanned volume, using the unfiltered radial velocity variances. Figure 15 (left panel) shows the estimate of $\sigma_{u}^{2}$ based on the unfiltered radial velocity variances of all bins without accounting for misalignment compared to $\sigma_{\text {cup }}^{2}$. The lidar-variance estimate is only $2 \%$ larger than the cup-anemometer value and the $R^{2}$ value is higher than that of any other comparison between cup-anemometer and lidar beam radial velocity variances (filtered or not).
In Fig. 15 (right panel) we show a similar comparison to the plot in the left panel but for the "filtered" $u$ variance, which was computed by reconstructing the $u$ and $v$ components, as described in Sect. 5.4, using the ZephIR measurements on the seven bins, but from the $18 \mathrm{~s}$ radial velocity measurements. The comparison with the filtered values shows poor agreement with a $50 \%$ underestimation of the variance by the ZephIR. However, reconstructed $u$ velocities from the $18 \mathrm{~s}$ radial velocities and averaged within $10 \mathrm{~min}$ periods compare well with the reconstructed values from the 10 min means; the mean bias is $0 \%$ and $R^{2}=0.999$ (not shown).

We also compare the lidar-derived $\sigma_{v, w}^{2}$ and $\overline{u^{\prime} w^{\prime}}$ values with the sonic-anemometer estimates; the biases are very high and $R^{2}$ values are very low (not shown). This is not surprising given the weight of the $\sigma_{v, w}^{2}$ and $\overline{u^{\prime} w^{\prime}}$ terms in Eq. (10) when using low $\varphi$ values. With this lidar configura- 

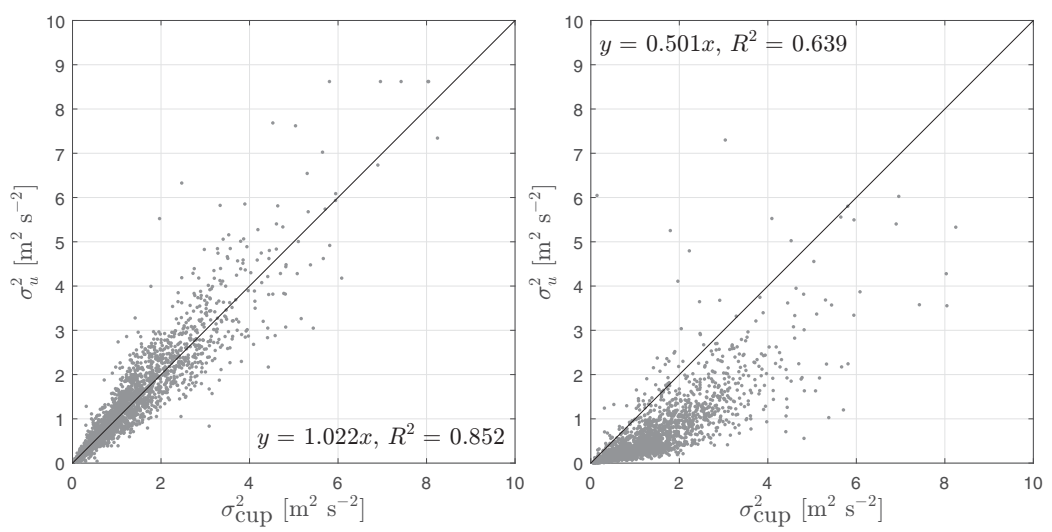

Figure 15. Comparison of the $80 \mathrm{~m}$ cup anemometer and the unfiltered (left) and filtered (right) $u$ variances from the ZephIR estimated under the assumption of homogeneous turbulence within the measurement volume (see text for details). We show a $1: 1$ line for guidance. Results of a linear regression through the origin and $R^{2}$ are also given.

tion, the reconstruction of the $v$ component from, for example, Eq. (14) is not sound either; the yaw misalignment based on both the Avent and ZephIR reconstructed $u$ and $v$ components shows poor agreement when compared to the difference between the wind-vane and the turbine-yaw $10 \mathrm{~min}$ signals.

We can also estimate $\sigma_{u}^{2}$ through a least-squares fit of Eq. (10) but using the unfiltered radial velocity variances of the horizontal bins (12 and 37) only and the comparison with $\sigma_{\text {cup }}^{2}$ shows similar results (bias of $3 \%$ and $R^{2}=0.842$ ). This indicates, firstly, the small but positive effect of adding the top and lower beams' variances, and secondly, that the contributions of other velocity components are not that significant for the estimation of $\sigma_{u}^{2}$ with the actual lidar configuration. Accounting for misalignment does not improve the variance comparison (the bias increases from 2 to $7 \%$ ).

\subsection{Effect of the noise filter on the lidar variances}

We also classify the $10 \mathrm{~min} 80 \mathrm{~m}$ cup anemometer variances and Avent radial velocity spectra into the classes given in Table 1, ensemble-average the spectra within each class, and compute the variance of each ensemble-average spectrum. The comparison of such variances, for each Avent beam, is illustrated in Fig. 16 (raw). We also show a similar comparison but for the noise-filtered Avent radial velocity ensemble-average spectra. Furthermore, we include the prediction $\sigma_{v_{\mathrm{r}}}^{2} / \sigma_{u}^{2}$ based on the Avent lidar configuration using the Mann model with fixed Mann parameters (same as those found in Sect. 5.2 using the ensemble-average sonicanemometer velocity spectra).

When the noise filter is applied, the ratio $\sigma_{v_{\mathrm{r}}}^{2} / \sigma_{\text {cup }}^{2}$ is well predicted by the Mann model. The largest difference is observed for beam 4 but this is because the noise filter highly reduces the variance for one particular class only. For beams 1 and 2, the Mann model predicts the same $\sigma_{v_{\mathrm{r}}}^{2} / \sigma_{\text {cup }}^{2}$ value as here we do not take into account lidar misalignment.

\subsection{Effect of atmospheric stability on turbulence spectra}

The ensemble-average sonic and Avent radial velocity spectra are used separately to extract two independent sets of Mann parameters for each of the atmospheric stability classes in Table 1 by fitting the sonic- and lidar-based LUTs computed through the use of Eqs. (3) and (6). Figure 17 shows the results of the two stability classes farthest apart (stabilities 1 and 5).

For the stability 1 class, the Mann model agrees well with the sonic velocity spectra and for stability 5 the differences between the model and the sonic-anemometer observations are larger, as expected, since the Mann model was developed for near-neutral atmospheric conditions. Both the sonic observations and the Mann model show the spectral peaks to move to higher wave numbers with increasing stability because the size of the turbulence eddies decreases with stability in agreement with the study of Peña et al. (2010a). The lidar radial velocity spectra also show similar features to the sonic-based spectra: higher normalized spectral densities for the most stable compared to the close to neutral class and spectral peaks that move to higher wave numbers with increasing stability. The former feature might be due to the way we normalize the spectra: we make use of the $76 \mathrm{~m}$ $u_{*}$ value instead of one close to the ground where surfacelayer scaling is more valid, particularly for stable conditions. The agreement of the Mann-model-based spectra also deteriorates with stability but the lidar-based LUT seems to follow the behavior of the radial velocity spectra for these two classes fairly well.

In Fig. 18, we show the results of the Mann parameters extracted from the ensemble-average sonic and lidar radial velocity spectra for all atmospheric stability classes. There is a slight decrease in $\Gamma$ with stability (based on the sonicanemometer data) and the lidar-based value closely follows the sonic-based one, with best agreement at the highest stability range. The sonic-based $\alpha \varepsilon^{2 / 3}$-parameter slightly de- 

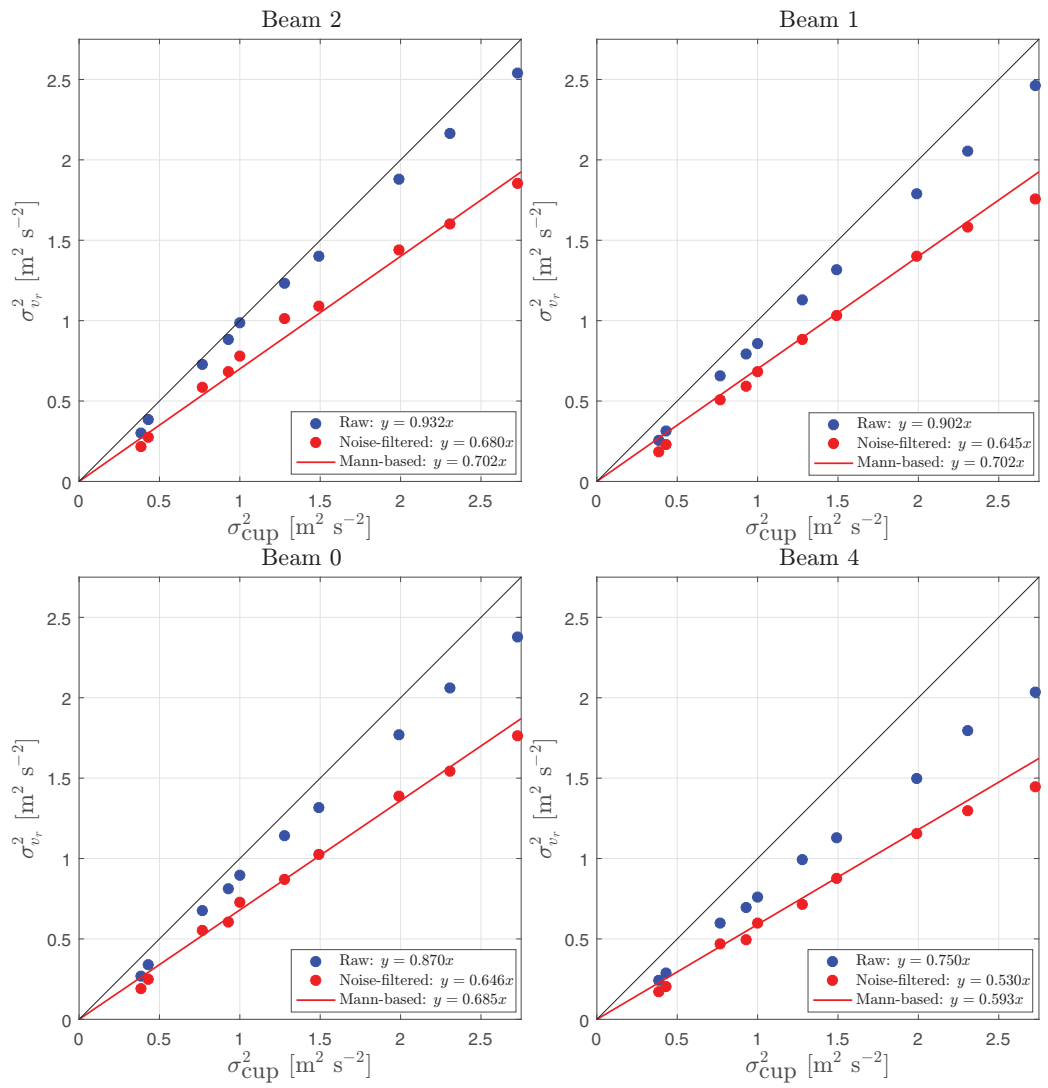

Figure 16. Comparison of the $80 \mathrm{~m}$ cup anemometer with the Avent radial velocity variances for different beams for the 10 turbulence classes (filled circles) in Table 1. Raw and noise-filtered data are shown as well as a $1: 1$ line (for guidance) and the prediction of the Avent filtered radial velocity variance based on the Mann model, using $\Gamma=3.00, L=35.38 \mathrm{~m}$, and $\beta=0^{\circ}$.

creases with stability and, for the near-neutral stability class, the lidar-based value is close to the sonic-based one. A similar feature is found for the $L$ parameter; both types of data show a very close value for near-neutral conditions and the sonic-based value slightly decreases with stability as expected. The increasing differences between the sonic- and the lidar-based $\alpha \varepsilon^{2 / 3}$ and $L$ parameters with stability are interconnected. We cannot expect to measure eddies below the size of the lidar probe volume, which in this case means that we are not able to accurately estimate the length scale when $L \lesssim z_{\mathrm{R}}$. This occurs already at the stability 3 class. These two Mann parameters are, in practical terms, scaling factors in the velocity spectra as seen from Eq. (4), and so an underestimation of $L$ generally leads to an overestimation of $\alpha \varepsilon^{2 / 3}$ when fitting the lidar-based LUT.

We also have to notice that when using this type of lidar configuration, we are extracting turbulence information from the radial velocity spectra of beams, whose spectral densities are rather close (since all beams measure a close to $u$ spectrum), whereas in the case of the sonic-anemometer observations we use three auto-spectra and a cross-spectrum that are relatively far apart in terms of spectral densities. This issue is discussed further in Sect. 7.

\subsection{Effect of wind speed on turbulence spectra}

We now perform a similar procedure as that in Sect. 6.4 but for each of the wind-speed classes in Table 1, and the results of the two wind-speed classes most far apart (speeds 1 and 5) are shown in Fig. 19. For the speed 1 class, the Mann model does not agree with the sonic velocity spectra as well as it does when compared to the speed 5 class, as expected, since the atmospheric conditions are closer to neutral for the latter class. Both the sonic-anemometer observations and the Mann model show spectral peaks that move to lower wave numbers with increasing wind speed because of the combined effect of stability and wind speed; the larger the turbulent eddies, the higher the wind speed and the lower the stability.

The lidar radial velocity spectra also show similar features to the sonic-based spectra; lower normalized spectral densities for the high-wind compared to the low-wind class and spectral peaks that move to lower wave numbers with increasing wind speed. The agreement of the Mann-modelbased spectra deteriorates with decreasing wind speed, but the lidar-based LUT also seems to follow the behavior of the radial velocity spectra for these two classes fairly well 

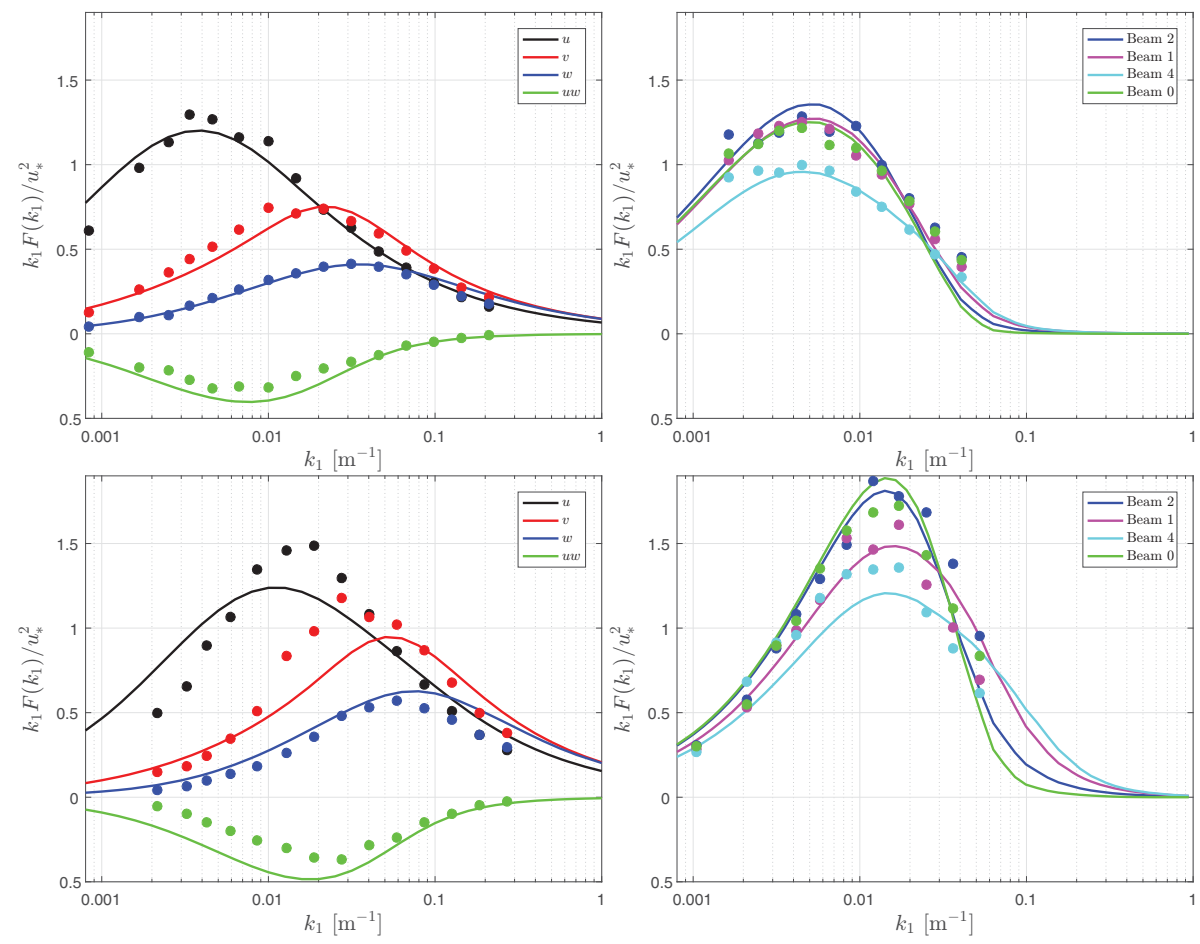

Figure 17. Normalized power spectra of the different velocity components based on the sonic-anemometer observations (left) and of the Avent radial velocity for different beams (right). The top panels show the results for the first stability range (stability 1) and the bottom panels for the last stability range (stability 5 ).
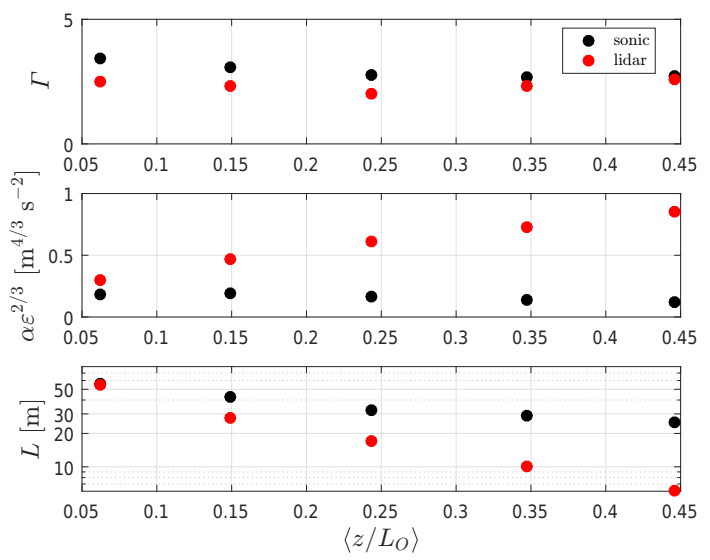

Figure 18. Mann parameters for a number of atmospheric-stability conditions (see Table 1) derived from sonic anemometer and lidar radial velocity spectra.

(similarly as it does when comparing spectra for the range of stability classes).

In Fig. 20, we show the results of the Mann parameters but for the wind-speed classes. Based on the sonic-anemometer data, $\Gamma$, is rather constant with wind speed, a behavior already observed by Peña et al. (2010a) for the same height and the lidar-based value agrees well with the sonic-based one for all wind-speed classes, particularly the two low wind- speed classes. Similar to the results from the atmosphericstability classes, the differences between the sonic- and the lidar-based $\alpha \varepsilon^{2 / 3}$ and $L$ parameters are larger than those for $\Gamma$, but for these wind-speed classes the $\alpha \varepsilon^{2 / 3}$ parameter does not differ largely under the classes where $L$ differs the most, i.e., speed classes 1 and 2, where the average conditions are very stable. Turbulence characteristics under these two classes are similar and $L$ is higher within speed 1 compared to the speed 2 class. The highest differences in the estimations of $L$ are also found for those classes in which $L \lesssim z_{\mathrm{R}}$ (speed classes 1-3).

\section{Discussion}

It is important to notice that some of the differences between turbulence statistics estimated from the sonic-, cupanemometer, and lidars' measurements are not only due to the way they probe the atmosphere but also because the lidar measurements are affected by optical and instrumental noise (and by the blades, hard targets, and fog, among other factors), the cup- and sonic-anemometers are inherently affected by flow distortion from the mast structure and by the instrument itself, which we do not take into account, and that there are differences in the heights of the measurements. For example, the axes of the lidars pointed close to hub height when the wind turbine was operating, and the $80 \mathrm{~m}$ cup and 

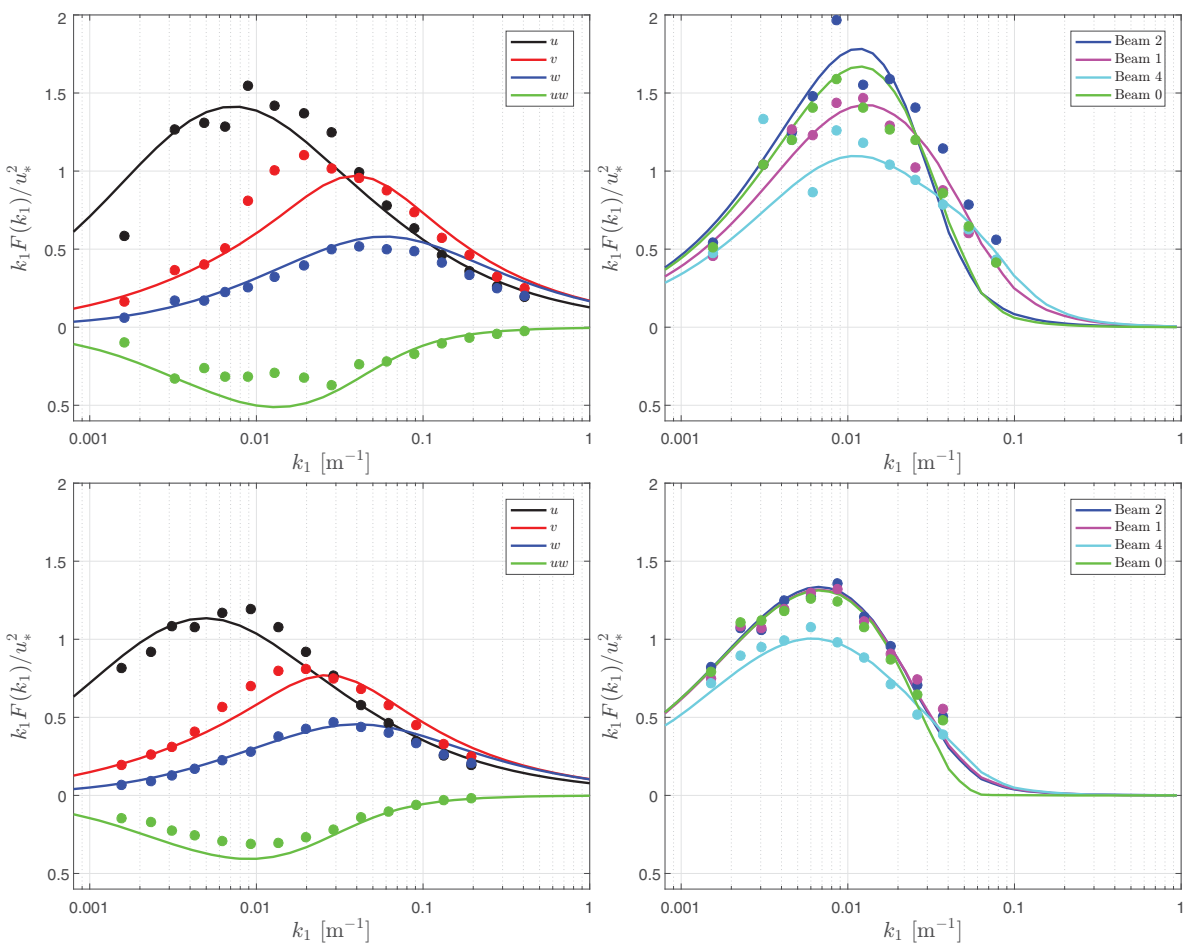

Figure 19. Similar to Fig. 17, but here the top panels show the results for the first wind-speed range (speed 1) and the bottom panels for the last wind-speed range (speed 5).

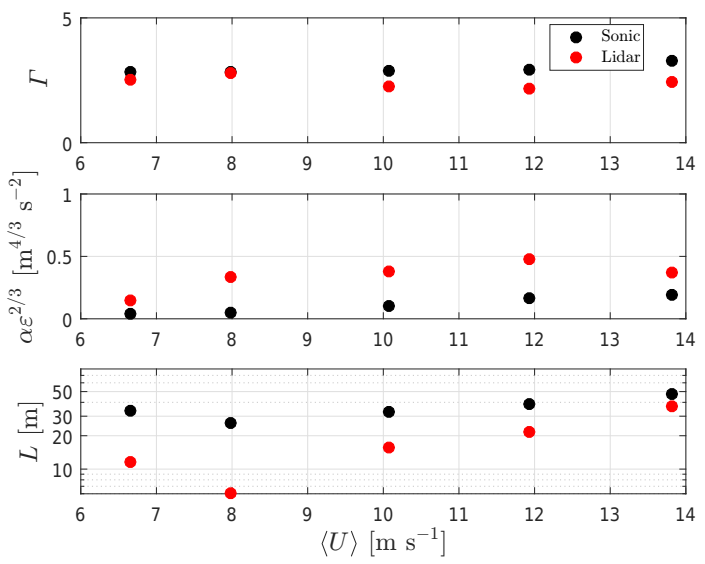

Figure 20. Mann parameters for a number of wind-speed ranges (see Table 1) derived from sonic anemometer and lidar radial velocity spectra.

sonic anemometer are 1.8 and $5.8 \mathrm{~m}$ below hub height, respectively. Also, the mast is $111 \mathrm{~m}$ from the range that we use to extract the lidar measurements when the wind is directly from the mast to the turbine. Wind speeds, variances, and velocity spectra from the mast and the lidars' selected range are expected to be comparable due to the topographic conditions of the site for the selected wind directions, but not equal. Further details regarding how cup anemometers, sonic anemometers and lidars measure turbulence are provided in
Kristensen (2000), Horst and Oncley (2006), and Sathe and Mann (2013), respectively.

We assume turbulence to be homogeneous within the lidar scanning area, both when extracting the Mann parameters and when studying the unfiltered turbulence. This is a rather simplistic assumption as shown in the study by Peña et al. (2010a), in which the Mann parameters are extracted from sonic-anemometer measurements at different heights. However, we expect that such an assumption results in turbulence parameters that are more representative of the turbine operation as they are estimated from measurements over a larger area.

In Sects. 6.4 and 6.5, we show normalized power spectra for the two most "extreme" classes in order to understand the spectra behavior for the changing atmospheric and windspeed conditions; spectra results for the other classes are not shown, but lie in between, as illustrated from the derived Mann parameters in Figs. 18 and 20. In Sect. 6.4, we mention that part of the problem of extracting the Mann parameters from the current lidar measurements is the small difference between the beams' radial velocity spectra, all being relatively close to the $u$ spectrum. The Mann model needs more than one-component spectra to fit the LUT to measurements/simulations, otherwise the Mann parameters are ill-determined.

We find very good agreement between the along-wind variance estimate of the ZephIR (when using the ensemble- 

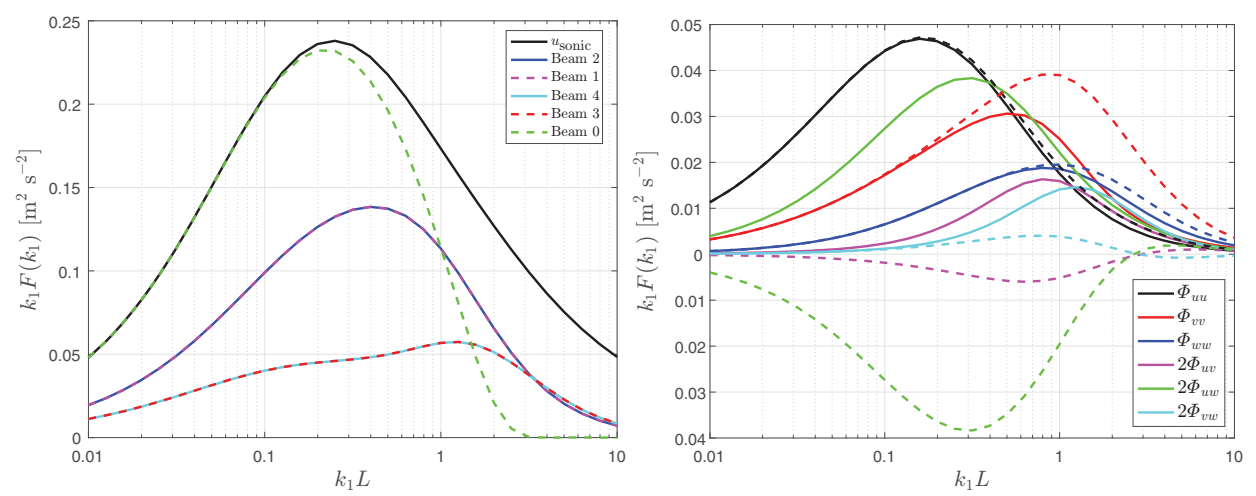

Figure 21. (Left panel) Pulsed lidar radial velocity spectra for different beams. (Right panel) Contributions of the spectral velocity tensor components to the lidar radial velocity spectrum for beams 2 (solid lines) and 3 (dashed lines). Values of $\beta=0^{\circ}, \varphi=60^{\circ}, \Gamma=3, \alpha \varepsilon^{2 / 3}=$ $0.1 \mathrm{~m}^{4 / 3} \mathrm{~s}^{-2}$, and $z_{\mathrm{R}} / L=0.5$ are used for the computation.

average Doppler radial velocity spectrum) and the cupanemometer measurement, but for the other velocitycomponent variances and co-variances, when compared to those from the sonic anemometer, the biases are too large. But, can we improve such estimates, e.g., increasing the cone angle $\varphi$ ? On the one hand, one can make the theoretical exercise of predicting $\sigma_{u, v, w}^{2}$ and $\overline{u^{\prime} w^{\prime}}$ from the Mann model (with a given set of Mann parameters). In parallel, we can use Eq. (6) with $z_{\mathrm{R}}=0$ to estimate the unfiltered $\sigma_{v_{\mathrm{r}}}^{2}$ for different beams and use Eq. (10) to estimate $\sigma_{u, v, w}^{2}$ and $\overline{u^{\prime} w^{\prime}}$ from the unfiltered beam variances. If we compare the former predicted with the latter estimated variances, e.g., using a four-beam lidar $\left(\theta=0,90,180\right.$, and $\left.270^{\circ}\right)$, with $\varphi=15^{\circ}$, the result for the $u$-variance is a $2 \%$ bias, whereas the $v$ and $w$ variances show biases larger than $50 \%$. The result for the $v$ and $w$ components improves when increasing $\varphi$; the biases for both components' variances are below $20 \%$ for $\varphi=60^{\circ}$ but the bias deteriorates for the $u$ variance with increasing $\varphi$. If a central beam is added and we are able to extract the unfiltered variance of this beam, i.e., $\sigma_{u}^{2}$, the comparisons are unbiased for all velocity components (no matter the value of $\varphi)$.

On the other hand, using a lidar with $\varphi=60^{\circ}$ increases the relative differences between the radial velocity spectra densities of the beams, e.g., with the current Avent configuration as it uses a central beam. Figure 21 shows that with such a cone angle, the central beam spectrum peaks close to the $u$ spectrum peak, and the lower beams peak at $\approx 20 \%$ of the $u$ spectrum peak (with $\varphi=15^{\circ}$, the lower beams peak at $\approx 75 \%$ of that of the central beam spectrum). This is mainly due to the large negative contribution of $\Phi_{u w}$ for the lower beams, as shown in Fig. 21 right panel. The difference between the $u$ and $w$ spectra is $\approx 60 \%$ only.

It is also important to highlight to the reader that wind turbine loads and power performance are directly impacted by turbulence, in particular $\sigma_{u}^{2}$. The latter affects the turbine's power output differently, depending on the wind speed
(Clifton and Wagner, 2014). The Mann parameters add value for understanding the behavior of loads but are not critical (Dimitrov et al., 2017). In this study we demonstrate that $\sigma_{u}^{2}$ can be estimated by FL nacelle lidars, and current research demonstrates that lidar-based $\sigma_{u}^{2}$ values reduce the gap between loads and power measurements, as well as simulations. It is difficult to compare our results with those from previous work on lidar turbulence measurements (Sathe et al., 2015; Newman et al., 2016). First, with a FL lidar we are able to point the beam in a direction close to the mean wind, whereas most lidars use beams pointing closer to the vertical wind component. Second, we do not need to reconstruct wind components to estimate variances, but the radial velocity spectrum and variance for each of the beams can be directly computed; this allows us to create a LUT useful to extract the Mann parameters.

\section{Conclusions}

We characterize turbulence using measurements from two types of forward-looking nacelle lidars that were mounted on the nacelle of a wind turbine. We compare such characteristics with those from sonic- and cup-anemometer measurements on a mast, which is $111 \mathrm{~m}$ from the lidar measurement range when the turbine and mast are aligned with the wind (thus this distance increases for other wind directions). By using information of the $10 \mathrm{~min}$ ensemble average Doppler radial velocity spectrum, we are able to estimate 10 min unfiltered radial-velocity variances of the beams of a $\mathrm{CW}$ lidar. These unfiltered beam variances are well predicted by the Mann model. Assuming homogeneous turbulence within the lidar scanned area, $\sigma_{u, v, w}^{2}$ and $\overline{u^{\prime} w^{\prime}}$ are estimated from the unfiltered beam variances; comparison with the 10 min cupanemometer variances reveals a $2 \%$ bias for the $u$ variance, whereas the biases are very high for the other velocity components. 
We divide the $10 \mathrm{~min}$ time series and the sonicanemometer and lidar beam radial velocity spectra into atmospheric-stability and wind-speed classes based on the mast measurements. Most of the conditions are stable and relatively windy. We observe that the pulsed lidar beam variances are affected by noise as clearly seen in the lidar radial velocity spectra. Therefore, we noise filter the lidar beam spectra, and the resulting variances show very good agreement with the prediction using the Mann and spatial averaging models.

We also extract the Mann parameters from sonicanemometer and lidar beam radial velocity spectra and intercompare them for each of the classes. Under high wind and near-neutral atmospheric conditions the agreement is good, and the differences increase with higher stability and lower wind speed, where the Mann model also has limitations fitting the sonic-anemometer velocity spectra. This is partly because increasing stability and decreasing wind speed results in turbulence length scales comparable to or lower than the length of the lidar probe volume. We suggest to improve lidar-based Mann-parameter estimations by increasing the lidars' cone angle, always keeping a central beam, which will also aid in the estimations of the non-wind-aligned velocity variances and covariances, although the flow homogeneity assumption becomes less valid.

Data availability. Turbine data are not publicly available because there is a non-disclosure agreement between the partners in the UniTTe project. Lidar and mast data can be requested from Rozenn Wagner at DTU Wind Energy (rozn@dtu.dk).

Competing interests. The authors declare that they have no conflict of interest.

Acknowledgements. Funding from Innovation Fund Denmark, grant number 1205-00024B, to the UniTTe project (http://www.unitte.dk) is acknowledged. We are grateful for the continuous technical support by Antoine Borraccino and Andrea Vignaroli, DTU Wind Energy.

Edited by: S. Aubrun

Reviewed by: W. Bierbooms and one anonymous referee

\section{References}

Aitken, M. L., Rhodes, M. E., and Lundquist, J. K.: Performance of a Wind-Profiling Lidar in the Region of Wind Turbine Rotor Disks, J. Atmos. Ocean. Tech., 29, 347-355, 2012.

Batchelor, G. K.: The theory of homogeneous turbulence, Cambridge University, Cambridge, 1953.

Bingöl, F., Mann, J., and Larsen, G. C.: Lidar Measurements of Wake Dynamics Part I: One Dimensional Scanning, Wind Energy, 13, 51-61, 2010.
Borraccino, A., Courtney, M., and Wagner, R.: Generic methodology for calibrating profiling nacelle lidars, Tech. Rep. DTU Wind Energy E-0086, Roskilde, Denmark, DTU Wind Energy, 2015.

Branlard, E., Pedersen, A. T., Mann, J., Angelou, N., Fischer, A., Mikkelsen, T., Harris, M., Slinger, C., and Montes, B. F.: Retrieving wind statistics from average spectrum of continuouswave lidar, Atmos. Meas. Tech., 6, 1673-1683, doi:10.5194/amt6-1673-2013, 2013.

Chougule, A., Mann, J., Segalini, A., and Dellwik, E.: Spectral tensor parameters for wind turbine load modeling from forested and agricultural landscapes, Wind Energy, 18, 469-481, 2015.

Clifton, A. and Wagner, R.: Accounting for the effect of turbulence on wind turbine power curves, J. Phys. Conf. Ser., 524, 012109, doi:10.1088/1742-6596/524/1/012109, 2014.

Dimitrov, N. and Natarajan, A.: Application of simulated lidar scanning patterns to load validation, Wind Energy, doi:10.1002/we.1992, 2016.

Dimitrov, N., Natarajan, A., and Mann, J.: Effects of normal and extreme turbulence spectral parameters on wind turbine loads, Renew. Energ., 101, 1180-1193, 2017.

Fleming, P. A., Scholbrock, A. K., Jehu, A., Davoust, S., Osler, E., Wright, A. D., and Clifton, A.: Field-test results using a nacelle-mounted lidar for improving wind turbine power capture by reducing yaw misalignment, J. Phys. Conf. Ser., 524, 012002, doi:10.1088/1742-6596/524/1/012002, 2014.

Hardesty, R. M., Korrell, J. A., and Hall, F. F.: Lidar measurements of wind velocity turbulence spectra encountered by a rotating turbine blade, Tech. Rep. DOE/RL/10236-81/1, NOAA/ERL/Wave Propagation Laboratory, Boulder, Colorado, USA, 1981.

Högström, U.: Non-dimensional wind and temperature profiles in the atmospheric surface layer: a re-evaluation, Bound.-Lay. Meteorol., 42, 55-78, 1988.

Horst, T. W. and Oncley, S. P.: Corrections to inertial-range power spectra measured by CSAT3 and Solent sonic anemometers, 1. Path-averaging errors, Bound.-Lay. Meteorol., 119, 375-395, 2006.

Kirchner, J. W.: Aliasing in $1 / f^{\alpha}$ noise spectra: Origins, consequences, and remedies, Phys. Rev. E, 71, 066110, doi:10.1103/PhysRevE.71.066110, 2005.

Kristensen, L.: Measuring higher-order moments with a cup anemometer, J. Atmos. Ocean. Tech., 17, 1139-1148, 2000.

Machefaux, E., Larsen, G., Troldborg, N., Hansen, K., Angelou, N., and Mikkelsen, T.: Investigation of wake interaction using full-scale lidar measurements and large eddy simulation, Wind Energy, 19, 1535-1551, 2016.

Mann, J.: The spatial structure of neutral atmospheric surface-layer turbulence, J. Fluid Mech., 273, 141-168, 1994.

Mann, J., Cariou, J.-P., Courtney, M. S., Parmentier, R., Mikkelsen, T., Wagner, R., Lindelöw, P., Sjöholm, M., and Enevoldsen, K.: Comparison of 3D turbulence measurements using three staring wind lidars and a sonic anemometer, Meteorol. Z., 18, 135-140, 2009.

Mann, J., Peña, A., Bingöl, F., Wagner, R., and Courtney, M. S.: Lidar scanning of momentum flux in and above the surface layer, J. Atmos. Ocean. Tech., 27, 959-976, 2010.

Mikkelsen, T., Angelou, N., Hansen, K., Sjöholm, M., Harris, M., Slinger, C., Hadley, P., Scullion, R., Ellis, G., and Vives, G.: A spinner-integrated wind lidar for enhanced wind turbine control, Wind Energy, 16, 625-643, 2013. 
Mizuno, T. and Panofsky, H. A.: The validity of Taylor's hypothesis in the atmospheric surface layer, Bound.-Lay. Meteorol., 9, 375$380,1975$.

Newman, J. F., Klein, P. M., Wharton, S., Sathe, A., Bonin, T. A., Chilson, P. B., and Muschinski, A.: Evaluation of three lidar scanning strategies for turbulence measurements, Atmos. Meas. Tech., 9, 1993-2013, doi:10.5194/amt-9-1993-2016, 2016.

Peña, A., Gryning, S.-E., and Mann, J.: On the length scale of the wind profile, Q. J. Roy. Meteor. Soc., 136, 2119-2131, 2010a.

Peña, A., Gryning, S.-E., and Hasager, C. B.: Comparing mixinglength models of the diabatic wind profile over homogeneous terrain, Theor. Appl. Climatol., 100, 325-335, 2010 b.

Peña, A., Hasager, C. B., Badger, M., Barthelmie, R. J., Bingöl, F., Cariou, J.-P., Emeis, S., Frandsen, S. T., Harris, M., Karagali, I., Larsen, S. E., Mann, J., Mikkelsen, T., Pitter, M., Pryor, S. C., Sathe, A., David Schlipf, C. S., and Wagner, R.: Remote sensing for wind energy, DTU Wind Energy report E-0084, DTU Wind Energy, Roskilde, Denmark, 2015.

Peña, A., Floors, R., Sathe, A., Gryning, S.-E., Wagner, R., Courtney, M. S., Larsén, X. G., Hahmann, A. N., and Hasager, C. B.: Ten years of boundary-layer and wind-power meteorology at Høvsøre, Denmark, Bound.-Lay. Meteorol., 158, 1-26, 2016.

Sathe, A. and Mann, J.: A review of turbulence measurements using ground-based wind lidars, Atmos. Meas. Tech., 6, 3147-3167, doi:10.5194/amt-6-3147-2013, 2013.
Sathe, A., Mann, J., Vasiljevic, N., and Lea, G.: A six-beam method to measure turbulence statistics using ground-based wind lidars, Atmos. Meas. Tech., 8, 729-740, doi:10.5194/amt-8-729-2015, 2015.

Schlipf, D., Haizmann, F., Cosack, N., Siebers, T., and Cheng, P. W.: Detection of Wind Evolution and Lidar Trajectory Optimization for Lidar-Assisted Wind Turbine Control, Meteorol. Z., 24, 565579, 2015.

Sjöholm, M., Mikkelsen, T., Mann, J., Enevoldsen, K., and Courtney, M.: Time series analysis of continuous-wave coherent Doppler Lidar wind measurements, Meteorol. Z, 18, 281-287, 2009.

Sonnenschein, C. M. and Horrigan, F. A.: Signal-to-Noise Relationships for Coaxial Systems that Heterodyne Backscatter from the Atmosphere, Appl. Optics, 10, 1600-1604, 1971.

Trujillo, J. J., Seifert, J. K., Würth, I., Schlipf, D., and Kühn, M.: Full-field assessment of wind turbine near-wake deviation in relation to yaw misalignment, Wind Energ. Sci., 1, 41-53, doi:10.5194/wes-1-41-2016, 2016.

Wagner, R., Pedersen, T. F., Courtney, M., Antoniou, I., Davoust, S., and Rivera, R. L.: Power curve measurement with a nacelle mounted lidar, Wind Energy, 17, 1441-1453, 2014.

Wyngaard, J. C.: Turbulence in the Atmosphere, Cambridge University, Cambridge, 2010. 